JOURNAL OF THE AMERICAN MATHEMATICAL SOCIETY

Volume 9, Number 4, October 1996

\title{
ON AN INNER PRODUCT IN MODULAR TENSOR CATEGORIES
}

\author{
ALEXANDER A. KIRILLOV, JR.
}

Dedicated to my father on his 60th birthday

\section{INTRODUCTION}

In this paper we study some properties of tensor categories that arise in 2dimensional conformal and 3-dimensional topological quantum field theory-socalled modular tensor categories. By definition, these categories are braided tensor categories with duality which are semisimple, have a finite number of simple objects and satisfy some non-degeneracy condition. Our main example of such a category is the reduced category of representations of a quantum group $U_{q} \mathfrak{g}$ in the case when $q$ is a root of unity (see $[\mathrm{AP}],[\mathrm{GK}]$ ).

The main property of such categories is that we can introduce a natural projective action of the mapping class group of any 2-dimensional surface with marked points on appropriate spaces of morphisms in this category (see [Tu]). This property explains the name "modular tensor category" and is crucial for establishing a relation with 3-dimensional quantum field theory and, in particular, for the construction of invariants of 3-manifolds (Reshetikhin-Turaev invariants).

In particular, for the torus with one puncture we get a projective action of the modular group $S L_{2}(\mathbb{Z})$ on any space of morphisms $\operatorname{Hom}(H, U)$, where $U$ is any simple object and $H$ is a special object which is an analogue of the regular representation (see [Lyu]). In the case $U=\mathbb{C}$ this action is well known: it is the action of a modular group on the characters of a corresponding affine Lie algebra. We study this action for arbitrary representation $U$; in particular, we show that this action is unitary with respect to a natural inner product on the space of intertwining operators.

In the special case $\mathfrak{g}=\mathfrak{s l}_{n}$ and $U$ a symmetric power of fundamental representation, this is closely related with Macdonald's theory. It was shown in the paper [EK3] (though we didn't use the word " $S$-matrix" there) that in this case the matrix coefficients of the matrix $S$ are some special values of Macdonald's polynomials of type $A_{n-1}$. Thus, the properties of $S$-matrix immediately yield a number of identities for values of Macdonald's polynomials at roots of 1 . In this case, the action of modular group is closely related with the difference Fourier transform defined in a recent paper of Cherednik ([Ch]). In particular, this shows that for $\mathfrak{g}=\mathfrak{s l}_{2}$

Received by the editors October 12, 1995 and, in revised form, November 20, 1995.

1991 Mathematics Subject Classification. Primary 81R50, 05E35, 18D10; Secondary 57M99.

Key words and phrases. Modular tensor categories, quantum groups at roots of 1, Macdonald polynomials. 
all matrix elements of the $S$-matrix can be written in terms of $q$-ultraspherical polynomials.

Unfortunately, a large part of this paper has to be devoted to recalling known facts about modular tensor categories and quantum groups at roots of unity; though these results are well known to experts, they are scattered in numerous papers, and some parts are not written anywhere at all. Thus, Sections 1 and 3 and a large part of Section 6 are expository.

The paper is organized as follows. In Section 1 we recall basic facts about modular tensor categories (MTC), in particular, the action of modular group and various symmetries of this action. In Section 2 we define an inner product on the space of intertwiners in modular tensor categories with some additional properties (hermitian MTC's), and prove that the action of modular group is unitary with respect to this inner product.

In Section 3 we recall, following Andersen, the construction of MTC from representations of quantum groups at roots of unity. In Section 4 we show that this category can be endowed with a natural hermitian structure.

Section 5 is devoted to a special case of the constructions above; namely, we let $\mathfrak{g}=\mathfrak{s l}_{n}$ and take $U$ to be a symmetric power of fundamental representation. We show that in this case the $S$-matrix can be written in terms of values of Macdonald's polynomials of type $A_{n-1}$ at roots of unity, which gives many identities for these special values. These expressions coincide with Cherednik's formulas for difference Fourier transform.

Sections 6 and 7 are devoted to further study of MTC's coming from quantum groups at roots of unity. In particular, we describe the Grothendieck ring of these categories (which is not new); we also give another description of the hermitian structure on them.

In subsequent papers we will apply the same construction to the modular tensor category arising from the affine Lie algebras, in which case it will give a modular invariant inner product on the space of conformal blocks.

\section{Modular tensor CATEgories}

In this section we review the main definitions relating to modular tensor categories. This section is completely expository and does not contain any new results. We start with a quick introduction to the notion of a ribbon category, introduced by Reshetikhin and Turaev ([RT1], [RT2]); we refer the reader to recent books by Kassel $([\mathrm{Kas}])$ and Turaev ([Tu] $)$ for detailed exposition.

Ribbon categories and graphs. A ribbon category is an additive category $\mathcal{C}$ with the following additional structures:

1. A bifunctor $\otimes: \mathcal{C} \times \mathcal{C} \rightarrow \mathcal{C}$ along with functorial associativity and commutativity isomorphisms:

$$
\begin{gathered}
a_{V_{1}, V_{2}, V_{3}}:\left(V_{1} \otimes V_{2}\right) \otimes V_{3} \rightarrow V_{1} \otimes\left(V_{2} \otimes V_{3}\right), \\
\check{R}_{V, W}: V \otimes W \rightarrow W \otimes V .
\end{gathered}
$$

2. A unit object $\mathbf{1} \in \operatorname{Obj} \mathcal{C}$ along with isomorphisms $\mathbf{1} \otimes V \rightarrow V, V \otimes \mathbf{1} \rightarrow V$. 
3. A notion of dual: for every object $V$ we have a (left) dual $V^{*}$ and homomorphisms

$$
\begin{aligned}
& e_{V}: V^{*} \otimes V \rightarrow \mathbf{1}, \\
& i_{V}: \mathbf{1} \rightarrow V \otimes V^{*} .
\end{aligned}
$$

4. Balancing, or a system of twists, i.e. functorial isomorphisms $\theta_{V}: V \rightarrow V$, satisfying the compatibility condition

$$
\theta_{V \otimes W}=\check{R}_{W, V} \check{R}_{V, W}\left(\theta_{V} \otimes \theta_{W}\right)
$$

These structures have to obey a number of properties, the list of which can be found in [Kas]. Using them, one can define functorial isomorphisms $\delta_{V}: V \rightarrow V^{* *}$ which are compatible with tensor products and unit objects. This, in particular, implies that for every $V$ we also have the right dual ${ }^{*} V$, which is canonically isomorphic to the left dual, and homomorphisms

$$
\begin{aligned}
& V \otimes^{*} V \rightarrow \mathbf{1}, \\
& \mathbf{1} \rightarrow{ }^{*} V \otimes V .
\end{aligned}
$$

In another terminology, ribbon categories are called braided monoidal rigid balanced categories (these words refer to the data we introduced in items 1-4 above, respectively).

Unless otherwise specified, we will assume that our category is abelian. We will also use the following theorem, due to Mac Lane: each ribbon category is equivalent to a strict one, i.e. such a category in which $\left(V_{1} \otimes V_{2}\right) \otimes V_{3}=V_{1} \otimes\left(V_{2} \otimes V_{3}\right)$ (not only isomorphic but is the same object!), and associativity morphism is the identity morphism; proof of this fact can be found in [Mac]. Unless otherwise specified, we only consider strict categories, and thus we can write tensor products of many objects without bothering about the parentheses.

Ribbon tensor categories admit a nice pictorial representation: if we have a directed tangle with braids labeled by objects of $\mathcal{C}$ and coupons labeled by morphisms, then we can assign to such a tangle a morphism in category $\mathcal{C}$ by certain rules - see [RT1], [RT2], [Tu] or [Kas]. The theorem proved by Reshetikhin and Turaev says that this morphism only depends on the isotopy class of the tangle; thus, we can prove identities about morphisms by manipulating with corresponding tangles. Also, note that if we replace a label $V$ of a certain braid by $V^{*}$ and reverse the direction of this braid, then we get the same morphism (up to canonical isomorphisms $V \simeq V^{* *}$ ).

For technical reasons, we will draw lines instead of ribbons; the only problem with that is that when establishing isotopy of graphs one must be careful to count the twists. Examples of tangles and corresponding operators and some identities are shown in Figure 1. Note that the operators act "from bottom to top", even though the arrows are oriented downwards. 


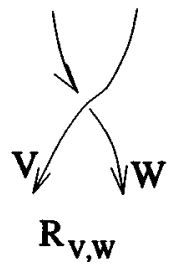

A. A. KIRILLOV, JR.

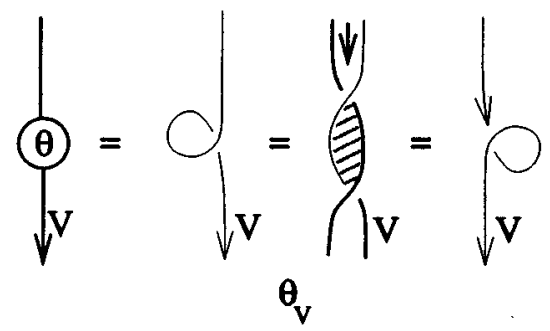

Figure 1a
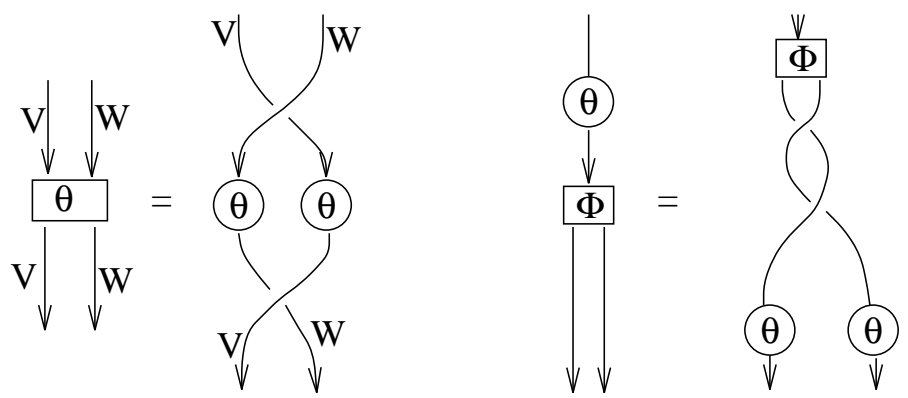

FiguRE 1b

Let us additionally assume that our category is semisimple, i.e.

1. It is defined over some field $\mathbb{K}$ : all the spaces of homomorphisms are finitedimensional vector spaces over $\mathbb{K}$.

2. Isomorphism classes of simple objects in $\mathcal{C}$ are indexed by elements of some set $I$; we will use the notation $X_{i}, i \in I$, for the corresponding simple objects, choosing the indexing so that $X_{0}=\mathbf{1}$. This implies that we have an involution * $: I \rightarrow I$ such that

$$
X_{i}^{*} \simeq X_{i^{*}}
$$

in particular, $0^{*}=0$.

3. "Schur's Lemma":

$$
\operatorname{Hom}\left(X_{i}, X_{j}\right)=\left\{\begin{array}{lc}
\mathbb{K}, & i=j, \\
0, & i \neq j .
\end{array}\right.
$$

4. Every object is completely reducible: every $V \in \operatorname{Obj} \mathcal{C}$ can be written in the form

$$
V=\bigoplus_{i \in I} N_{i} X_{i}
$$

where $N_{i} \in \mathbb{Z}_{+}$, and the sum is finite (i.e., almost all $N_{i}=0$ ).

Remark. In fact, these axioms are abundant: for example, $\operatorname{Hom}\left(X_{i}, X_{j}\right)=0$ for $i \neq j$ can be deduced from other axioms, see $[\mathrm{Tu}]$. 
Semisimplicity is a very restrictive requirement; it implies a lot of properties. For example, we can define the multiplicity coefficients $N_{i j}^{k}$ by

$$
X_{i} \otimes X_{j}=\bigoplus N_{i j}^{k} X_{k},
$$

and then we have the following obvious properties:

$$
\begin{gathered}
N_{i j}^{k}=\operatorname{dim} \operatorname{Hom}\left(X_{i} \otimes X_{j}, X_{k}\right)=\operatorname{dim} \operatorname{Hom}\left(X_{i} \otimes X_{j} \otimes X_{k}^{*}, \mathbf{1}\right), \\
N_{i j}^{k}=N_{j i}^{k}=N_{i k^{*}}^{j^{*}}=N_{i^{*} j^{*}}^{k^{*}}, \\
N_{i j}^{0}=\delta_{i j^{*}} .
\end{gathered}
$$

For an object $V \in \operatorname{Obj} \mathcal{C}$ we define its dimension $\operatorname{dim} V \in \mathbb{K}$ by the following picture:

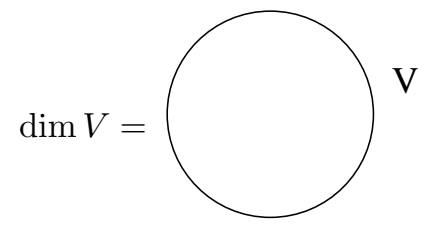

More generally, for a morphism $f \in \operatorname{Hom}(V, V)$ we define its trace $\operatorname{Tr} f$ by the following picture:

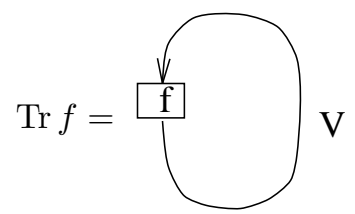

When the objects of a category are vector spaces over some field, the dimension and trace defined above are usually called "quantum dimension" (respectively, "quantum trace") to distinguish from ordinary dimension and trace.

Lemma 1.1. 1. $\operatorname{dim} V^{*}=\operatorname{dim} V, \operatorname{dim} \mathbf{1}=1$.

2. $\operatorname{dim} V \otimes W=\operatorname{dim} V \cdot \operatorname{dim} W$.

Action of modular group. As before, we assume that we have a semisimple ribbon category $\mathcal{C}$ with simple objects $X_{i}, i \in I$. Define the numbers $s_{i j} \in \mathbb{K}$ by the following picture:

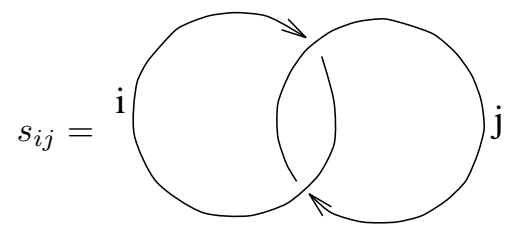

(From now on, we will often label strands of tangles by the indices $i \in I$ meaning $X_{i}$.)

Proposition 1.2.

$$
\begin{aligned}
s_{i j}=s_{j i} & =s_{i^{*} j^{*}}=s_{j^{*} i^{*}}, \\
s_{i 0} & =\operatorname{dim} X_{i} .
\end{aligned}
$$


Definition 1.3. A semisimple ribbon category $\mathcal{C}$ is called modular if it satisfies the following properties:

1. It has only a finite number of simple objects: $|I|<\infty$.

2. The matrix $s=\left(s_{i j}\right)_{i, j \in I}$, where $s_{i j}$ is defined by (1.4), is invertible.

We will give an example of a modular category later.

Remark. In fact, many authors (for example, Turaev) impose weaker conditions, not necessarily requiring semisimplicity in our sense. We are only interested in the simplest case; thus the above definition is absolutely sufficient for our purposes. We refer the reader to $[\mathrm{Ke}]$ for a discussion of the non-semisimple case.

Proposition 1.4. In a modular category, we have $\operatorname{dim} X_{i} \neq 0$ and

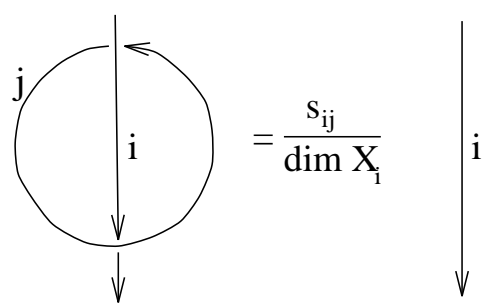

The name "modular" is justified by the fact that in this case we can define a projective action of the modular group $S L_{2}(\mathbb{Z})$ on certain objects in our category, which we will show below. To the best of my knowledge, this construction first appeared (in rather vague terms) in papers of Moore and Seiberg ([MS2], [MS4]); later it was formalized by Lyubashenko ([Lyu $]$ ) and others. Our exposition follows the book of Turaev.

The appearance of modular group in tensor categories may seem mysterious; however, there is a simple geometrical explanation, based on the fact that to each modular tensor category one can associate a 2+1-dimensional Topological Quantum Field Theory. This also shows that in fact we have an action of the mapping class group of any closed oriented 2-dimensional surface on the appropriate objects in MTC. This is the key idea of the book $[\mathrm{Tu}]$.

From now on, let us adopt the following convention: if some (closed) strand on a picture is left unlabeled, then we assume summation over all labels $i \in I$, each taken with the weight $\operatorname{dim} X_{i}$. Then we have the following propositions, proof of which (not too difficult) can be found in [Tu].

Let us define the numbers $\theta_{i} \in \mathbb{K}$ by

$$
\theta_{X_{i}}=\theta_{i} \operatorname{Id}_{X_{i}}
$$

then it is easy to see that $\theta_{i}=\theta_{i^{*}}, \theta_{0}=1$.

Proposition 1.5. We have the following identities:
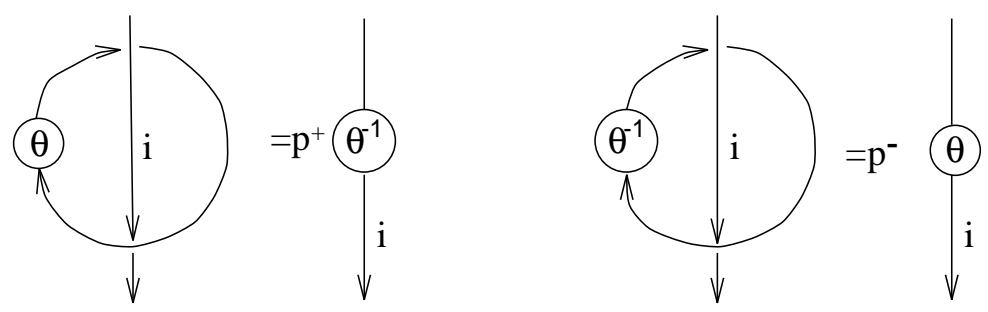
where

$$
p^{ \pm}=\sum_{i \in I} \theta_{i}^{ \pm 1}\left(\operatorname{dim} X_{i}\right)^{2}
$$

Also,

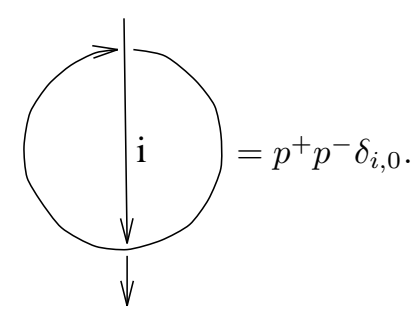

Corollary 1.6. 1 .

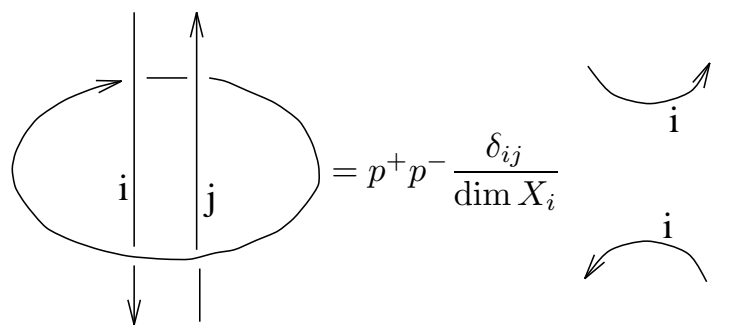

2.

$$
p^{+} p^{-}=\sum\left(\operatorname{dim} X_{i}\right)^{2}
$$

Define the matrices $t=\left(t_{i j}\right)$ and $c=\left(c_{i j}\right)$ ("charge conjugation matrix") by

$$
\begin{aligned}
t_{i j} & =\delta_{i j} \theta_{i}, \\
c_{i j} & =\delta_{i j^{*}} .
\end{aligned}
$$

Then it is easy to deduce from Proposition 1.5 the following theorem:

Theorem 1.7. The matrices $s, t$ defined above satisfy the following relations:

$$
\begin{gathered}
s^{2}=p^{+} p^{-} c, \\
(s t)^{3}=p^{+} s^{2}, \\
s^{2} t=t s^{2},
\end{gathered}
$$

where $p^{ \pm}$are defined by (1.8).

It is convenient to renormalize these matrices. Namely, let us assume that the following fractional powers exist in $\mathbb{K}$ :

$$
\begin{gathered}
D=\sqrt{p^{+} p^{-}}=\sqrt{\sum_{i \in I}\left(\operatorname{dim} X_{i}\right)^{2}}, \\
\zeta=\left(p^{+} / p^{-}\right)^{1 / 6}
\end{gathered}
$$

(we choose the roots so that $D \zeta^{3}=p^{+}$). It follows from non-degeneracy of $s$ that $D, \zeta \neq 0$. 
Define renormalized matrices

$$
\tilde{s}=\frac{s}{D}, \quad \tilde{t}=\frac{t}{\zeta} .
$$

Then Theorem 1.7 is rewritten in the following form:

$$
\begin{gathered}
\tilde{s}^{2}=c, \\
(\tilde{s} \tilde{t})^{3}=\tilde{s}^{2}, \\
\tilde{s}^{2} \tilde{t}=\tilde{t} \tilde{s}^{2} .
\end{gathered}
$$

Since $c^{2}=1$, this shows that $\tilde{s}, \tilde{t}$ give a representation of the modular group $S L_{2}(\mathbb{Z})$. Recall that $S L_{2}(\mathbb{Z})$ is generated by the elements

$$
S=\left(\begin{array}{cc}
0 & -1 \\
1 & 0
\end{array}\right), \quad T=\left(\begin{array}{ll}
1 & 1 \\
0 & 1
\end{array}\right)
$$

satisfying the defining relations $(S T)^{3}=S^{2}, S^{2} T=T S^{2}, S^{4}=1$.

Now, let us define the following object in $\mathcal{C}$ :

$$
H=\bigoplus_{i \in I} X_{i} \otimes X_{i}^{*}
$$

We assume that we have fixed isomorphisms $X_{i}^{*} \simeq X_{i^{*}}$ as in (1.1), and thus we can also write $H$ as $\bigoplus X_{i} \otimes X_{i}^{*}$ or $\bigoplus X_{i}^{*} \otimes X_{i}$. Note that since $\left(X_{i} \otimes X_{i}^{*}\right)^{*} \simeq X_{i} \otimes X_{i}^{*}$, we have an isomorphism $H \simeq H^{*}$.

Definition 1.8. Define $S, T, C \in$ End $H$ as follows: $S=\bigoplus S_{i j}, S_{i j}: X_{j} \otimes X_{j^{*}} \rightarrow$ $X_{i} \otimes X_{i^{*}}$, and similarly for $T, C$, where $S_{i j}, T_{i j}, C_{i j}$ are given by:

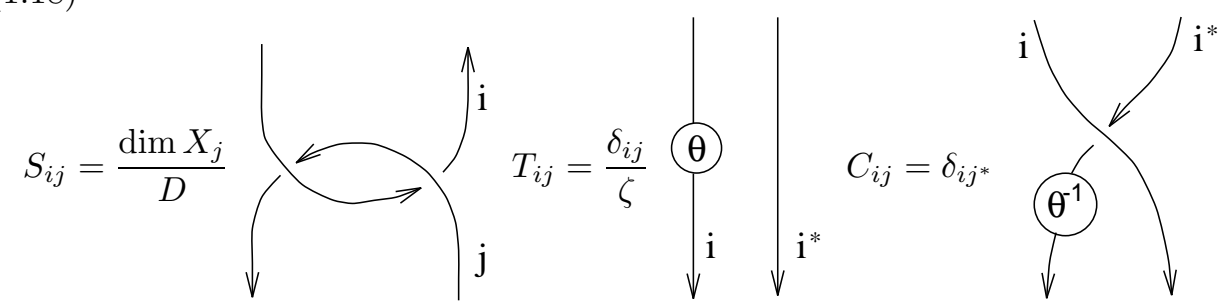

Theorem 1.9. The morphisms $S, T, C$ defined above satisfy the following relations:

$$
\begin{gathered}
S^{2}=C, \\
S^{4}=C^{2}=\theta_{H}^{-1}, \\
(S T)^{3}=S^{2}, \\
S^{2} T=T S^{2} .
\end{gathered}
$$

Proof. Follows from Proposition 1.5 and Corollary 1.6.

We cannot say that $S, T$ give a projective representation of the modular group in $H$, since $\theta_{H}$ is not a constant. However, it is true if we restrict them to an isotypic component of $H$. Equivalently, let us fix a simple object $U$ in our category and consider the space

$$
\operatorname{Hom}(H, U)=\bigoplus \operatorname{Hom}\left(X_{i} \otimes X_{i}^{*}, U\right) .
$$

This is a vector space over $\mathbb{K}$, and $\left.\theta_{H}\right|_{\operatorname{Hom}(H, U)}=\theta_{U} \operatorname{Id}_{\operatorname{Hom}(H, U)}$. 
Theorem 1.10. Define the maps $S_{U}, T_{U}: \operatorname{Hom}(H, U) \rightarrow \operatorname{Hom}(H, U)$ by

$$
\begin{aligned}
& S_{U}: \Phi \mapsto \Phi S, \\
& T_{U}: \Phi \mapsto \Phi T .
\end{aligned}
$$

Then $S_{U}, T_{U}$ satisfy the following relations:

$$
\begin{gathered}
S_{U}^{4}=\theta_{U}^{-1}, \\
T_{U} S_{U}^{2}=S_{U}^{2} T_{U}, \\
\left(S_{U} T_{U}\right)^{3}=S_{U}^{2},
\end{gathered}
$$

and thus give a projective representation of the group $S L_{2}(\mathbb{Z})$ in $\operatorname{Hom}(H, U)$.

Example. Let $U=\mathbf{1}$ be the unit object in $\mathcal{C}$. Then we have a canonical identification $\operatorname{Hom}\left(X_{i}^{*} \otimes X_{i}, \mathbf{1}\right) \simeq \mathbb{K}$, and thus we have a canonical basis $\chi_{i} \in \operatorname{Hom}(H, \mathbf{1})$. In this case, the action of the modular group defined in Theorem 1.10 in the basis $\chi_{i}$ is given by $\tilde{s}, \tilde{t}$ defined by (1.15).

The following result, which is a reformulation of a theorem of Vafa, is also worth mentioning here (though we won't use it):

Theorem 1.11. In any modular tensor category (regardless of the base field) the numbers $\theta_{i}, \zeta$ (see $\left.(1.6),(1.14)\right)$ are roots of unity.

This theorem was proved by Vafa (see [Vaf]) in the context of conformal field theory. However, his proof only uses some relation in the mapping class group of $n$ punctured sphere and the action of $S L_{2}(\mathbb{Z})$. Both groups act in arbitrary modular tensor category: the action of $S L_{2}(\mathbb{Z})$ was discussed above, and the action of the mapping class group can be defined as well (see [Tu, V.4]). Thus, the same proof is valid in arbitrary MTC. Note that for MTC's coming from Conformal Field Theory, we have $\zeta=e^{2 \pi \mathrm{i} c / 24}$, where $c$ is the central charge of the action of Virasoro algebra, and the theorem above implies that $c$ is rational, which is why these theories are called rational.

Hermitian categories. We will also need the notion of hermitian category: this definition and all the properties we are citing are due to Turaev (see [Tu]). Let us assume that $\mathcal{C}$ is a ribbon category which is defined over the ground field $\mathbb{K}$ equipped with an involution $x \mapsto \bar{x}$; our basic examples of such an involution will be $\mathbb{K}=\mathbb{C}$ with usual complex conjugation, and $\mathbb{K}=\mathbb{C}(q), \bar{q}=q^{-1}$. We say that $\mathcal{C}$ is hermitian if for every pair of objects $V, W$ we have an involutive map $-\operatorname{Hom}(V, W) \rightarrow \operatorname{Hom}\left(V^{*}, W^{*}\right)$ such that $\overline{f+g}=\bar{f}+\bar{g}, \overline{\alpha f}=\bar{\alpha} \bar{f}$ for any $\alpha \in \mathbb{K}, \overline{f g}=\bar{f} \bar{g}, \overline{f \otimes g}=\bar{g} \otimes \bar{f}$, and $\overline{\mathrm{Id}_{V}}=\operatorname{Id}_{V^{*}}$. Note that since we have a canonical identification $\operatorname{Hom}\left(V^{*}, W^{*}\right) \simeq \operatorname{Hom}(W, V)$, we could as well consider $\bar{f}$ as an element of $\operatorname{Hom}(W, V)$. This involution must satisfy certain compatibility properties, namely:

$$
\begin{gathered}
\bar{\theta}_{V}=\theta_{V^{*}}^{-1}, \\
\check{R}_{V, W}=\left(\check{R}_{V^{*}, W^{*}}\right)^{-1}, \\
\overline{e_{V}}=e_{V}\left(1 \otimes \delta_{V}^{-1}\right): V^{*} \otimes V^{* *} \rightarrow \mathbf{1},
\end{gathered}
$$

where $e_{V}$ is the canonical morphism $V^{*} \otimes V \rightarrow \mathbf{1}$. Then it can be shown that geometrically this involution corresponds to reflection: if $f$ is a morphism corresponding to the ribbon graph $\Gamma$, then $\bar{f}$ corresponds to the graph $\bar{\Gamma}$ obtained by 
reflection of $\Gamma$ in the plane $x=1$ (we assume that the graph is drawn in the projection to $x, y$-plane) and changing each label $V$ to $V^{*}$. Note that this operation changes the orientation of $\mathbb{R}^{3}$.

If $\mathcal{C}$ is a hermitian modular category, then it follows from the above geometric interpretation of bar conjugation that we have the following identities:

$$
\begin{aligned}
\overline{s_{i j}} & =s_{i j^{*}}, \\
\overline{\theta_{i}} & =\theta_{i}^{-1} .
\end{aligned}
$$

Thus,

$$
\begin{gathered}
\overline{\operatorname{dim} V}=\operatorname{dim} V, \\
\overline{p^{+}}=p^{-}, \quad \overline{p^{-}}=p^{+} .
\end{gathered}
$$

Therefore, $p^{+} p^{-}$is "real" and $p^{+} / p^{-}$is "unitary": $\overline{p^{+} p^{-}}=p^{+} p^{-}, \overline{p^{+} / p^{-}}=$ $\left(p^{+} / p^{-}\right)^{-1}$. We assume that $D$ and $\zeta$ (see (1.14)) can also be chosen "real" and "unitary" respectively:

$$
\bar{D}=D, \quad \bar{\zeta}=\zeta^{-1}
$$

Obviously, it is so if $\mathbb{K}=\mathbb{C}$, since in this case $D^{2}=\sum\left(\operatorname{dim} X_{i}\right)^{2} \in \mathbb{R}_{+}$, and $|\zeta|=1$.

Proposition 1.12. The matrices $\tilde{s}, \tilde{t} \in M a t_{|I|}(\mathbb{K})$ are "unitary", i.e. satisfy $X X^{*}$ $=1$, where $\left(X^{*}\right)_{i j}=\overline{X_{j i}}$.

Proof. Obvious from (1.16), (1.23).

Similar statement holds in a more general case:

Proposition 1.13. The operators $S, T \in$ End $H$ satisfy the following properties:

$$
\begin{gathered}
\bar{S}=S C^{-1}, \\
\bar{T}=T^{-1} .
\end{gathered}
$$

Thus, both $S$ and T satisfy

$$
X \bar{X}=\operatorname{Id}_{H}
$$

Proof. It is obvious for $T$; as for $S$, we need to prove that $\left(S C^{-1}\right)_{i j}$ is given by the following picture:

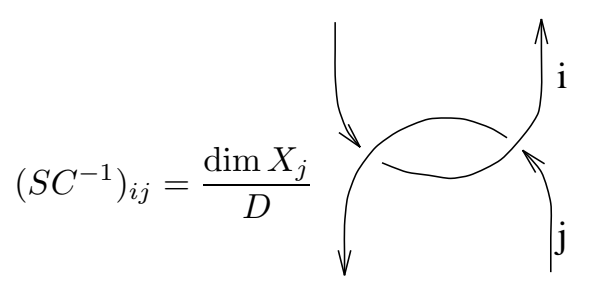

which easily follows from the definitions.

In the next chapter we will show how Proposition 1.13 can be interpreted as "unitarity" with respect to a certain inner product in $H$. 


\section{INNER PRODUCT ON MORPHISMS}

In this section we define an inner product on spaces of morphisms in a hermitian MTC; this definition is due to Turaev. We also show that the action of modular group defined in Section 1 is unitary with respect to this action; the same applies to associativity and commutativity isomorphisms (when rewritten in terms of Hom spaces). These results are new.

As before, we assume that $\mathcal{C}$ is a modular category; we keep all the notations and conventions of Section 1.

Definition 2.1. Let $V, W$ be objects from $\mathcal{C}$. Assume that $\operatorname{dim} V \neq 0, \operatorname{dim} W \neq 0$ and that there exist $\sqrt{\operatorname{dim} V}, \sqrt{\operatorname{dim} W}$ in $\mathbb{K}$. Define the pairing

$$
\operatorname{Hom}(V, W) \otimes \operatorname{Hom}\left(V^{*}, W^{*}\right) \rightarrow \mathbb{K}
$$

as follows: if $\Phi_{1} \in \operatorname{Hom}(V, W), \Phi_{2} \in \operatorname{Hom}\left(V^{*}, W^{*}\right)$, then let

$$
\left\langle\Phi_{1}, \Phi_{2}\right\rangle=\frac{1}{(\operatorname{dim} V \operatorname{dim} W)^{1 / 2}}
$$

Obviously, this pairing is symmetric.

Examples. 1. Let $V=W$. Then $\left\langle\operatorname{Id}_{V}, \operatorname{Id}_{V^{*}}\right\rangle=1$ (this justifies the choice of normalization in Definition 2.1).

2. Consider intertwiners of the form $\Phi_{1}: X_{i} \otimes X_{j} \rightarrow X_{k}, \Phi_{2}: X_{j^{*}} \otimes X_{i^{*}} \rightarrow X_{k^{*}}$. Then Definition 2.1 allows one to define a pairing between them provided that we have chosen identifications $X_{i^{*}} \simeq X_{i}^{*}$, etc. Note that in this case the dimension $\operatorname{dim}\left(X_{i} \otimes X_{j} \otimes X_{k}^{*}\right)$ is non-zero automatically.

If $\mathcal{C}$ is a hermitian category, then we define a "hermitian" inner product on $\operatorname{Hom}(V, W)$ by

$$
\left(\Phi_{1}, \Phi_{2}\right)=\left\langle\Phi_{1}, \overline{\Phi_{2}}\right\rangle
$$

as usual, we will denote $\|\Phi\|^{2}=(\Phi, \Phi)$. It is easy to see that this inner product satisfies the usual relations

$$
\begin{gathered}
\left(\alpha \Phi_{1}, \Phi_{2}\right)=\alpha\left(\Phi_{1}, \Phi_{2}\right), \quad \alpha \in \mathbb{K}, \\
\left(\Phi_{2}, \Phi_{1}\right)=\overline{\left(\Phi_{1}, \Phi_{2}\right)} .
\end{gathered}
$$

Lemma 2.2 ([Tu]). In a hermitian modular category, the inner product given by (2.2) is non-degenerate.

Remark. Obviously, the definition of the pairing (and thus, of the inner product) works as well in a ribbon category without the assumption of modularity; however, in this case it is not always true that this inner product is non-degenerate.

Lemma 2.3. Let $\Phi_{1}, \Phi_{1}^{\prime}: V_{1} \otimes V_{2} \rightarrow X_{i}, \Phi_{2}, \Phi_{2}^{\prime}: X_{i} \otimes V_{3} \rightarrow U$ be morphisms in a hermitian modular category, and let $\Psi=\Phi_{2}\left(\Phi_{1} \otimes 1\right), \Psi^{\prime}=\Phi_{2}^{\prime}\left(\Phi_{1}^{\prime} \otimes 1\right) \in$ $\operatorname{Hom}\left(V_{1} \otimes V_{2} \otimes V_{3}, U\right)$. Then

$$
\left(\Psi, \Psi^{\prime}\right)=\left(\Phi_{1}, \Phi_{1}^{\prime}\right)\left(\Phi_{2}, \Phi_{2}^{\prime}\right)
$$


Proof. The proof follows from the identity

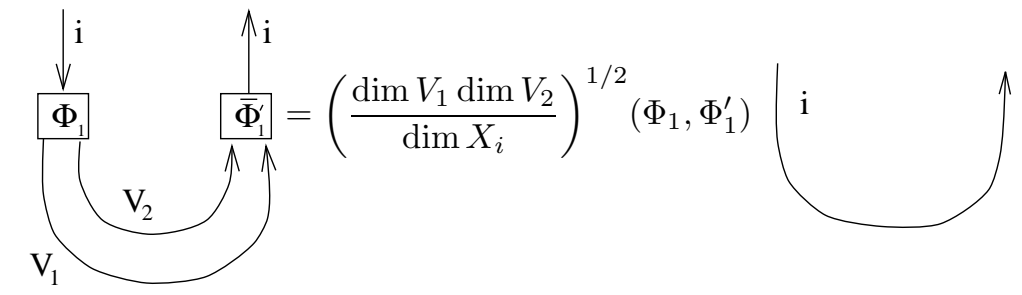

We can rewrite commutativity and associativity isomorphisms in terms of Hom spaces, which gives us isomorphisms

$$
\begin{gathered}
\check{R}: \operatorname{Hom}\left(V_{1} \otimes V_{2}, U\right) \rightarrow \operatorname{Hom}\left(V_{2} \otimes V_{1}, U\right), \\
\alpha: \bigoplus_{i \in I} \operatorname{Hom}\left(V_{1} \otimes V_{2}, X_{i}\right) \otimes \operatorname{Hom}\left(X_{i} \otimes V_{3}, U\right) \rightarrow \\
\bigoplus_{i \in I} \operatorname{Hom}\left(V_{1} \otimes X_{i}, U\right) \otimes \operatorname{Hom}\left(V_{2} \otimes V_{3}, X_{i}\right) .
\end{gathered}
$$

Theorem 2.4. In a hermitian modular tensor category, the associativity and commutativity isomorphisms (2.4) are unitary, i.e. preserve the inner product (2.2). The same is true for the isomorphism $\operatorname{Hom}\left(V_{1} \otimes V_{2}, V_{3}\right) \simeq \operatorname{Hom}\left(V_{1} \otimes V_{2} \otimes{ }^{*} V_{3}, \mathbf{1}\right)$.

Proof. Unitarity of associativity isomorphism follows from Lemma 2.3; unitarity of commutativity follows from the following picture:

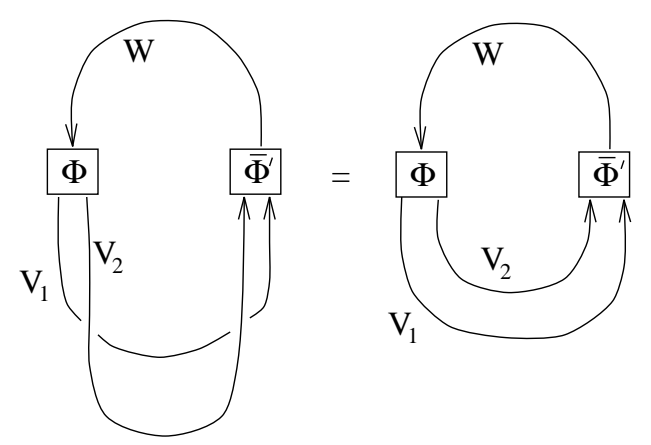

Unitarity of the last isomorphism is obvious.

In particular, (2.2) gives a natural inner product on each space $\operatorname{Hom}\left(X_{i} \otimes X_{i}^{*}, U\right)$, and thus - by taking the direct sum over all $i$ - on $\operatorname{Hom}(H, U)$. (Note that because of the normalizations, the inner product $\left(\Phi_{1}, \Phi_{2}\right)$ depends on whether we consider $\Phi_{1}, \Phi_{2}$ as intertwiners $X_{i} \otimes X_{i}^{*} \rightarrow U$ or $H \rightarrow U$. We will always use the former choice, i.e. consider $\Phi$ as morphisms $X_{i} \otimes X_{i}^{*} \rightarrow U$.)

Theorem 2.5. Let $U$ be an irreducible object. Then the inner product in the space $\operatorname{Hom}(H, U)$ defined by $(2.2)$ is invariant under the projective action of the modular group on $\operatorname{Hom}(H, U)$, which was defined in Theorem 1.10. 
Proof. In view of the identities $S_{U} \overline{S_{U}}=1, T_{U} \overline{T_{U}}=1$ (Proposition 1.13), it suffices to show that $\left(\Phi_{1} S, \Phi_{2}\right)=\left(\Phi_{1}, \Phi_{2} \bar{S}\right)$, or, equivalently, $\left\langle\Phi_{1} S, \overline{\Phi_{2}}\right\rangle=\left\langle\Phi_{1}, \overline{\Phi_{2}} S\right\rangle$, and similarly for $T$. For $T$ this is obvious; for $S$, it follows from the following picture:
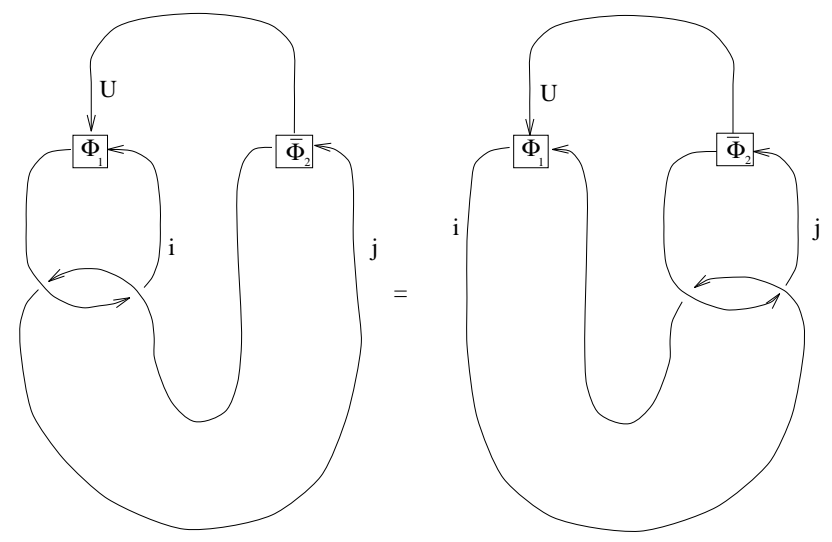

\section{Quantum groups at Roots of Unity}

In this section we recall the known results on construction of modular categories from representations of quantum groups at roots of unity, following the papers of Andersen ([A], [AP]) (which, in turn, are based on the work of Lusztig ([L1]-[L4], see also [L5])), and Gelfand-Kazhdan ([GK]). Again, this section is completely expository.

General facts on quantum groups. Here we give the main definitions from the theory of quantum groups; they are well known, and we refer the reader to the original papers by Drinfeld and Jimbo or to Lusztig's book [L5] for details, giving here the bare minimum - mostly to fix notations.

Let $\mathfrak{g}$ be a simple Lie algebra over $\mathbb{C}$. We use the standard notations for the Cartan subalgebra, roots, weights etc.; we also denote by $\theta$ the highest root of $\mathfrak{g}$. We denote by $($, ) invariant bilinear form in $\mathfrak{h}$ normalized so that $(\theta, \theta)=2$, and by $(,)^{\prime}=m\left(\right.$, ) the form normalized so that $(\alpha, \alpha)^{\prime}=2$ for short roots; equivalently, it is specified by the conditions $d_{i}=\left(\alpha_{i}, \alpha_{i}\right)^{\prime} / 2 \in \mathbb{Z}_{+}, \operatorname{gcd} d_{i}=1$. Thus, $m=1$ for simply-laced root systems, $m=2$ for root systems of $B, C, F$ types and $m=3$ for $G_{2}$.

By definition, the corresponding quantum group $U_{q} \mathfrak{g}$ is an algebra over the field $\mathbb{C}_{q}=\mathbb{C}\left(q^{1 / 2 N}\right)$, where $N=|P / Q|$ (fractional powers are necessary to define braiding) with generators $e_{i}, f_{i}, q^{h}, h \in \frac{1}{2} Q^{\vee} \subset \mathfrak{h}, i=1, \ldots, r$, and relations

$$
\begin{gathered}
q^{0}=1, \quad q^{a+b}=q^{a} q^{b}, \\
q^{h} f_{j} q^{-h}=q^{-\left\langle h, \alpha_{j}\right\rangle} f_{j}, \\
q^{h} e_{j} q^{-h}=q^{\left\langle h, \alpha_{j}\right\rangle} e_{j}, \\
{\left[e_{i}, f_{j}\right]=\delta_{i j} \frac{q^{d_{i} h_{i}}-q^{-d_{i} h_{i}}}{q^{d_{i}}-q^{-d_{i}}},}
\end{gathered}
$$




$$
\begin{aligned}
& \sum_{n=0}^{1-a_{i j}} \frac{(-1)^{n}}{[n]_{i} !\left[1-a_{i j}-n\right]_{i} !} e_{i}^{n} e_{j} e_{i}^{1-a_{i j}-n}=0 \\
& \sum_{n=0}^{1-a_{i j}} \frac{(-1)^{n}}{[n]_{i} !\left[1-a_{i j}-n\right]_{i} !} f_{i}^{n} f_{j} f_{i}^{1-a_{i j}-n}=0
\end{aligned}
$$

where $h_{i}=\alpha_{i}^{\vee} \in \mathfrak{h}$ and

$$
[n]_{i}=\frac{q^{n d_{i}}-q^{-n d_{i}}}{q^{d_{i}}-q^{-d_{i}}}, \quad[n]_{i} !=[1]_{i} \ldots[n]_{i} .
$$

This is a Hopf algebra with the following comultiplication, counit and antipode:

$$
\begin{gathered}
\Delta e_{i}=e_{i} \otimes q^{d_{i} h_{i} / 2}+q^{-d_{i} h_{i} / 2} \otimes e_{i}, \Delta f_{i}=f_{i} \otimes q^{d_{i} h_{i} / 2}+q^{-d_{i} h_{i} / 2} \otimes f_{i}, \\
\Delta q^{h}=q^{h} \otimes q^{h}, \\
\epsilon\left(q^{h}\right)=1, \epsilon\left(e_{i}\right)=\epsilon\left(f_{i}\right)=0, S\left(e_{i}\right)=-q^{d_{i}} e_{i}, S\left(f_{i}\right)=-q^{-d_{i}} f_{i}, S\left(q^{h}\right)=q^{-h} .
\end{gathered}
$$

As is well-known, this algebra is quasitriangular: there exists a "universal Rmatrix" $\mathcal{R}$ which is an element of a certain completion of $U_{q} \mathfrak{g} \otimes U_{q} \mathfrak{g}$ such that for every pair of finite-dimensional representations $V, W$ the operator

$$
\check{R}_{V, W}=P \circ \pi_{V} \otimes \pi_{W}(\mathcal{R}): V \otimes W \rightarrow W \otimes V
$$

is an isomorphism of representations. Here $P$ is the transposition: $P v \otimes w=w \otimes v$. Also, it is known that $\mathcal{R}$ has the following form:

$$
\begin{gathered}
\mathcal{R}=q^{\sum a_{i} \otimes a_{i}} \mathcal{R}^{*}, \quad \mathcal{R}^{*} \in U^{+} \hat{\otimes} U^{-}, \\
(\epsilon \otimes 1)\left(\mathcal{R}^{*}\right)=(1 \otimes \epsilon)\left(\mathcal{R}^{*}\right)=1 \otimes 1,
\end{gathered}
$$

where $a_{i}$ is an orthonormal basis in $\mathfrak{h}$ with respect to $(,)^{\prime}$. As we said, $\mathcal{R}$ does not lie in the tensor square of $U_{q} \mathfrak{g}$ but in a certain completion of it; however, for any pair of finite-dimensional representations $V, W$ of $U_{q} \mathfrak{g}$ the operator $\pi_{V} \otimes \pi_{W}(\mathcal{R})$ is well-defined (this is where we need fractional powers of $q$ in the definition of $\mathbb{C}_{q}$ ).

Remark. This definition differs from Lusztig's one by a slightly different choice of generators and, more importantly, by the change $v=q^{-1}$.

We recall (see $[\mathrm{Kas}],[\mathrm{Tu}]$ ) that the category $\operatorname{Rep} U_{q} \mathfrak{g}$ of finite-dimensional representations of $U_{q} \mathfrak{g}$ is a semisimple ribbon category (in the sense of definitions of Section 1) defined over $\mathbb{C}_{q}$. Its simple objects are irreducible highest-weight modules $V_{\lambda}, \lambda \in P^{+}$. Note that if $\lambda \in P^{+}$, then $V_{\lambda}^{*} \simeq V_{\lambda^{*}}$, where $\lambda^{*}=-w_{0}(\lambda), w_{0}$ being the longest element of the Weyl group $W$. In this category, the balancing map is such that $\theta_{V_{\lambda}}=q^{(\lambda, \lambda+2 \rho)^{\prime}}$, and the isomorphism $\delta_{V}: V \rightarrow V^{* *}$ is given by $q^{2 \rho}$, where $\rho$ is considered as an element of $\mathfrak{h}$ using the identification given by $(,)^{\prime}$; thus, $q^{2 \rho} v=q^{2(\rho, \lambda)^{\prime}} v$ if $v$ has weight $\lambda$. This implies that the quantum dimension of a module is given by $\operatorname{dim}_{q} V=\operatorname{Tr}_{V}\left(q^{2 \rho}\right)$. In particular, if $V_{\lambda}$ is irreducible, then $\operatorname{dim}_{q} V_{\lambda}=\chi_{\lambda}\left(q^{2 \rho}\right)$, where $\chi_{\lambda} \in \mathbb{C}[P]$ is the character of $V_{\lambda}$, and we use the following convention: for $f=\sum a_{\lambda} e^{\lambda} \in \mathbb{C}_{q}[P]$ we let

$$
f\left(q^{\mu}\right)=\sum a_{\lambda} q^{(\lambda, \mu)^{\prime}} .
$$

It follows from the Weyl formula that

$$
\operatorname{dim}_{q} V_{\lambda}=\chi_{\lambda}\left(q^{2 \rho}\right)=\frac{\sum_{w \in W}(-1)^{l(w)} q^{2(\rho, w(\lambda+\rho))^{\prime}}}{\delta\left(q^{2 \rho}\right)}=\frac{\delta\left(q^{2(\lambda+\rho)}\right)}{\delta\left(q^{2 \rho}\right)},
$$


where $\delta$ is the Weyl denominator:

$$
\delta=\sum_{W}(-1)^{l(w)} e^{w(\rho)}=\prod_{\alpha \in R^{+}}\left(e^{\alpha / 2}-e^{-\alpha / 2}\right) .
$$

Representations of $U_{q} \mathfrak{g}$ at roots of unity and the category $\mathcal{C}(\mathfrak{g}, \varkappa)$. Let $A=\mathbb{Z}\left[q^{1 / 2 N}, q^{-1 / 2 N}\right]$, and let $U$ be the $A$-subalgebra of $U_{q} \mathfrak{g}$ generated by $e_{i}^{n} /[n]$ !, $f_{i}^{n} /[n] !, q^{h}$ (see [L2]). For an arbitrary non-zero number $\varepsilon \in \mathbb{C}$ define

$$
U_{\varepsilon}=U \otimes_{A} \mathbb{C}
$$

where $\mathbb{C}$ is endowed with an $A$-module structure by $q \mapsto \varepsilon$.

Our goal is to construct a certain subquotient of the category of finite-dimensional representations of $U_{\varepsilon}$ in the case when $\varepsilon$ is a root of unity:

$$
\varepsilon=e^{\pi \mathrm{i} / m \varkappa},
$$

where $m$ is as before and $\varkappa \in \mathbb{Z}_{+}$. In this paper we always assume that $\varkappa \geq h^{\vee}$, where $h^{\vee}$ is the dual Coxeter number for $\mathfrak{g}$. By definition, we let $\varepsilon^{a}=e^{\pi \mathrm{i} a / m \varkappa}$ for $a \in \frac{1}{2 N} \mathbb{Z}$, and as before, for $f=\sum a_{\lambda} e^{\lambda} \in \mathbb{C}[P]$ we let

$$
f\left(\varepsilon^{\mu}\right)=\sum a_{\lambda} \varepsilon^{(\lambda, \mu)^{\prime}}=\sum a_{\lambda} e^{\pi \mathrm{i}(\lambda, \mu) / \varkappa} .
$$

Recall (see [L1]) that due to the fact that $U_{\varepsilon}$ contains divided powers, we have a notion of weight subspace, and weight subspaces are indexed by $P$ (not by $P / 2 \varkappa P !$ ). Let $\operatorname{Rep} U_{\varepsilon}$ be the category of finite-dimensional representations of $U_{\varepsilon}$ with weight decomposition.

Theorem 3.1. Rep $U_{\varepsilon}$ has a natural structure of ribbon category over $\mathbb{C}$.

Proof. This follows from a general result due to Lusztig ([L5, Chapter 32]); we refer the reader to $[\mathrm{KL} 4, \S 37]$ for details.

Let $V_{\lambda}$ be the irreducible finite-dimensional module over $U_{q} \mathfrak{g}$ with highest weight $\lambda \in P^{+}$, and let $v_{\lambda}$ be the highest weight vector in it. $V_{\lambda}$ admits a $U$-structure: we can consider $U$-submodule $U v_{\lambda} \subset V_{\lambda}$. Thus, we can define a module over $U_{\varepsilon}$ which we denote by $V_{\lambda}^{\varepsilon}$ (sometimes we will also denote it by $V_{\lambda}$ ):

$$
V_{\lambda}^{\varepsilon}=\left(U v_{\lambda}\right) \otimes_{A} \mathbb{C} \text {. }
$$

These modules are usually called Weyl modules and are not necessarily irreducible (see below).

Define the open and closed alcoves $C, \bar{C}$ by

$$
\begin{gathered}
C=\left\{\lambda \in P^{+} \mid\left\langle\lambda+\rho, \theta^{\vee}\right\rangle<\varkappa\right\}, \\
\bar{C}=\left\{\lambda \in P \mid\left\langle\lambda+\rho, \alpha_{i}^{\vee}\right\rangle \geq 0,\left\langle\lambda+\rho, \theta^{\vee}\right\rangle \leq \varkappa\right\} .
\end{gathered}
$$

Note that $C$ is preserved by the involution $\lambda \mapsto \lambda^{*}=-w_{0}(\lambda)$.

We will denote by $\Gamma$ the affine wall of $\bar{C}$ :

$$
\Gamma=\left\{\lambda \in \mathfrak{h}^{*} \mid\left\langle\lambda+\rho, \theta^{\vee}\right\rangle=\varkappa\right\} .
$$

Note that $\bar{C}$ is the fundamental domain for the shifted action of the affine Weyl group $\widetilde{W}$ of level $\varkappa$. Recall that the shifted action is defined by $w \cdot \lambda=w(\lambda+\rho)-\rho$, and $\widetilde{W}=W \ltimes \varkappa Q^{\vee}$, where $Q^{\vee}$ is considered as a lattice in $\mathfrak{h}^{*}$ using the identification $\mathfrak{h} \simeq \mathfrak{h}^{*}$ given by the form $($,$) ; under this identification Q^{\vee} \subset Q$.

Now we can formulate the main result on the reducibility of Weyl modules: 
Lemma 3.2. For $\lambda \in \bar{C} \cap P^{+}$, the Weyl modules $V_{\lambda}$ are irreducible.

In general, Weyl modules are not irreducible. However, it is easy to see that for every $\lambda \in P^{+}$there exists a unique irreducible highest-weight module $L_{\lambda}$, and that every irreducible module in $\operatorname{Rep} U_{\varepsilon}$ is of the form $L_{\lambda}, \lambda \in P^{+}$. Thus, every module from $\operatorname{Rep} U_{\varepsilon}$ has a composition series with factors of the form $L_{\lambda}$. In particular, the same is true for $V_{\lambda}$, and the multiplicities (conjecturally) express in terms of Kazhdan-Lusztig polynomials associated with the affine Weyl group $\widetilde{W}$ (see [L6, Section 9]).

Our goal is to extract from this highly non-trivial category of representations some semisimple part. This was first done by Reshetikhin and Turaev in the case $\mathfrak{g}=\mathfrak{s l}_{2}$ and by Andersen $([\mathrm{A}],[\mathrm{AP}])$ in the general case. We briefly sketch the main steps here.

Let us call a module $V \in \operatorname{Rep} U_{\varepsilon}$ tilting if both $V$ and $V^{*}$ have a composition series with the factors isomorphic to $V_{\lambda}$. Let $\mathcal{T}$ be the full subcategory of $\operatorname{Rep} U_{\varepsilon}$ consisting of tilting modules. This category is closed under taking dual representations (obvious) and under tensor product (see $[\mathrm{A}],[\mathrm{AP}]$ ), and thus is a ribbon category. Note that in particular the modules $V_{\lambda}, \lambda \in C$, are tilting.

However, the category of tilting modules is still too large, and thus we need further reduce it. This is done by factorization over negligible modules.

For every finite-dimensional module $V$ over $U_{\varepsilon}$ we define its $\operatorname{dimension} \operatorname{dim}_{\varepsilon} V$ by the same formula as for $U_{q} \mathfrak{g}$; in particular, for the highest-weight module $V_{\lambda}^{\varepsilon}$ we have $\operatorname{dim}_{\varepsilon} V_{\lambda}^{\varepsilon}=\chi_{\lambda}\left(\varepsilon^{2 \rho}\right)$. Simple calculation gives the following result:

Lemma 3.3. Let $\lambda \in P^{+}$. Then

$$
\operatorname{dim}_{\varepsilon} V_{\lambda}=0 \Longleftrightarrow(\lambda+\rho, \alpha) \in \varkappa \mathbb{Z} \text { for some } \alpha \in R^{+} .
$$

In particular, if $\lambda \in C$, then $\operatorname{dim}_{\varepsilon} V_{\lambda} \neq 0$, and if $\lambda \in \Gamma \cap P^{+}$, then $\operatorname{dim}_{\varepsilon} V_{\lambda}=0$ (recall that $\Gamma$ denotes the affine wall of $\bar{C}$ ).

Let us call a tilting module $V$ negligible if for every $f \in$ End $V$ we have $\operatorname{Tr}_{\varepsilon}(f)=$ 0 . The following simple lemma describes the properties of negligible modules:

Lemma 3.4 ([A]). 1. An indecomposable module $V \in \operatorname{Rep} U_{\varepsilon}$ is negligible iff $\operatorname{dim}_{\varepsilon} V=0$.

2. $V$ is negligible iff $V^{*}$ is negligible.

3. If $\lambda \in C$, then $V_{\lambda}$ is not a direct summand of a negligible module; equivalently, for every $\lambda \in C$ and a negligible module $Z$ the composition

$$
V_{\lambda} \stackrel{f}{\rightarrow} Z \stackrel{g}{\rightarrow} V_{\lambda}
$$

is equal to zero for any morphisms $f, g$.

4. If $V$ is a negligible module, then $V \otimes V^{\prime}$ is negligible for any $V^{\prime} \in \operatorname{Rep} U_{\varepsilon}$.

The following key theorem is due to Andersen:

Theorem 3.5. Every tilting module $V$ can be written in the form

$$
V=\left(\bigoplus_{\lambda \in C} n_{\lambda} V_{\lambda}\right) \oplus Z
$$

for some negligible tilting module $Z$ and uniquely defined non-negative integers $n_{\lambda}$. 
In particular, this theorem implies that for $\lambda, \mu \in C$ we can write

$$
V_{\lambda} \otimes V_{\mu}=\left(\bigoplus_{\nu \in C} N_{\lambda \mu}^{\nu} V_{\nu}\right) \oplus Z,
$$

for some $N_{\lambda \mu}^{\nu} \in \mathbb{Z}_{+}$and $Z$ as above.

In the case $\mathfrak{g}=\mathfrak{s l}_{2}$ this theorem was proved by Reshetikhin and Turaev.

This theorem allows us to define the modular category with simple objects $V_{\lambda}, \lambda \in C$, as follows. Recall that $\mathcal{T}$ is the full subcategory of tilting modules in $\operatorname{Rep} U_{\varepsilon}$. Let $\mathcal{T}^{\text {neg }} \subset \mathcal{T}$ be the full subcategory of negligible tilting modules. We want to define the quotient category $\mathcal{C}=\mathcal{T} / \mathcal{T}^{\text {neg }}$; this construction is due to $[\mathrm{GK}]$, and we briefly sketch it below.

Let $V_{1}, V_{2} \in \mathcal{T}$. We call a morphism $f: V_{1} \rightarrow V_{2}$ negligible if it can be presented in the form $f=g h$ for some $h: V_{1} \rightarrow Z, g: Z \rightarrow V_{2}$, where $Z$ is a negligible module. We denote negligible morphisms from $V$ to $W$ by $\operatorname{Hom}^{\text {neg }}(V, W)$.

Definition 3.6. The quotient category $\mathcal{C}(\mathfrak{g}, \varkappa)=\mathcal{C}$ is defined as follows:

$$
\begin{gathered}
\operatorname{Ob} \mathcal{C}=\mathrm{Ob} \mathcal{T}, \\
\operatorname{Hom}_{\mathcal{C}}(V, W)=\operatorname{Hom}_{U_{\varepsilon}}(V, W) / \operatorname{Hom}_{U_{\varepsilon}}^{\text {neg }}(V, W) .
\end{gathered}
$$

It follows from Lemma 3.4 that if $f$ is a negligible morphism, then for any morphism $g$ the composition $f g$ is also negligible; the same applies to $g f, f^{*}$ and $f \otimes g$. Therefore, compositions, tensor products and duals of morphisms are welldefined and thus $\mathcal{C}(\mathfrak{g}, \varkappa)$ is a ribbon category. Obviously, in this category every negligible module is isomorphic to the zero module. Thus, Theorem 3.5 implies that every object in $\mathcal{C}$ is isomorphic to a direct sum

$$
V=\bigoplus_{\lambda \in C} n_{\lambda} V_{\lambda}
$$

for some unique collection of non-negative integers $n_{\lambda}$. In particular, we have the following isomorphism in $\mathcal{C}$ :

$$
V_{\lambda} \otimes V_{\mu} \simeq \bigoplus_{\nu \in C} N_{\lambda \mu}^{\nu} V_{\nu}
$$

where the numbers $N_{\lambda \mu}^{\nu}$ are the same as in (3.16).

Warning: in general, $N_{\lambda \mu}^{\nu} \neq \operatorname{dim}_{\operatorname{Hom}}\left(V_{\lambda} \otimes V_{\mu}, V_{\nu}\right)$. Instead, we have the following result:

Lemma 3.7. Let $\lambda, \mu, \nu \in C$. Then

$$
\operatorname{Hom}_{U_{q} \mathfrak{g}}\left(V_{\lambda} \otimes V_{\mu}, V_{\nu}\right) "=" \operatorname{Hom}_{U_{\varepsilon}}\left(V_{\lambda}^{\varepsilon} \otimes V_{\mu}^{\varepsilon}, V_{\nu}^{\varepsilon}\right) .
$$

The equality should be understood in the following sense: we can define some intertwining operators $\Phi_{i}$ which are defined over $A=\mathbb{Z}\left[q^{ \pm 1 / 2 N}\right]$ such that $\Phi_{i}$, considered as intertwining operators over $\mathbb{C}_{q}$ (respectively, over $\mathbb{C}$ ), form a basis in $\operatorname{Hom}_{U_{q} \mathfrak{g}}\left(V_{\lambda} \otimes V_{\mu}, V_{\nu}\right)$ (respectively, in $\left.\operatorname{Hom}_{U_{\varepsilon}}\left(V_{\lambda}^{\varepsilon} \otimes V_{\mu}^{\varepsilon}, V_{\nu}^{\varepsilon}\right)\right)$-compare with definition (3.13) of $V_{\lambda}^{\varepsilon}$.

Proof. It follows from the fact that one can write an explicit formula for such an intertwiner involving only the inverse of the Shapovalov form (see, for example, [EK3]). Since Shapovalov form is non-degenerate in both $V_{\lambda}$ (as a matrix with entries from $\mathbb{C}_{q}$ ) and in $V_{\lambda}^{\varepsilon}$ (as a matrix with complex entries), this proves the lemma. 
$\mathcal{C}(\mathfrak{g}, \varkappa)$ as a modular category. Let us summarize the properties of the category $\mathcal{C}$ :

Proposition 3.8. 1 . The category $\mathcal{C}(\mathfrak{g}, \varkappa)$ is semisimple, and its simple objects are precisely $\left\{V_{\lambda}\right\}_{\lambda \in C}$.

2. For any object $V \in \mathcal{C}(\mathfrak{g}, \varkappa)$ we have $\operatorname{dim}_{\varepsilon} V \in \mathbb{R}_{>0}$.

3. This category has a natural structure of ribbon category, inherited from $\operatorname{Rep} U_{\varepsilon}$.

4. The matrices $s_{i j}, t_{i j}$ defined by (1.4), (1.12) for the category $\mathcal{C}(\mathfrak{g}, \varkappa)$ are given by

$$
\begin{gathered}
t_{\lambda \mu}=\delta_{\lambda \mu} \varepsilon^{(\lambda, \lambda+2 \rho)^{\prime}} \\
s_{\lambda \mu}=\chi_{\lambda}\left(\varepsilon^{-2(\mu+\rho)}\right) \operatorname{dim}_{\varepsilon} V_{\mu}^{\varepsilon}=\frac{\sum_{w \in W}(-1)^{l(w)} \varepsilon^{-2(w(\lambda+\rho), \mu+\rho)}}{\delta\left(\varepsilon^{-2 \rho}\right)},
\end{gathered}
$$

where $\chi_{\lambda} \in \mathbb{C}[P]^{W}$ is the character of the module $V_{\lambda}, \delta$ is the Weyl denominator and we use convention (3.12).

Proof. (1) was already discussed; (2) follows from the Weyl formula for $\operatorname{dim}_{\varepsilon} V_{\lambda}$ and (1); (3) is obvious. Formula (3.18) is also very well known and can be deduced from the diagonal part of the $R$-matrix (see, for example, arguments in [EK3]).

Theorem 3.9. The matrix $s$ defined by (3.18) is non-degenerate, and thus $\mathcal{C}(\mathfrak{g}, \varkappa)$ is a modular category. Also, in this case the numbers $D=\sqrt{p^{+} p^{-}}=\sqrt{\sum\left(\operatorname{dim}_{\varepsilon} V_{\lambda}\right)^{2}}$, $\zeta=\left(p^{+} / p^{-}\right)^{1 / 6}(c f .(1.14))$ are given by

$$
\begin{gathered}
D=\frac{\sqrt{\left|P / \varkappa Q^{\vee}\right|}}{\mathrm{i}^{\left|R^{+}\right|} \delta\left(\varepsilon^{-2 \rho}\right)}=\frac{\sqrt{\left|P / \varkappa Q^{\vee}\right|}}{\prod_{\alpha \in R^{+}} 2 \sin \frac{(\alpha, \rho)}{\varkappa} \pi}, \\
\zeta=\varepsilon^{\frac{\varkappa-h^{\vee}}{h^{\vee}}(\rho, \rho)^{\prime}}=e^{2 \pi \mathrm{i} c / 24}, \quad c=\frac{\left(\varkappa-h^{\vee}\right) \operatorname{dim} \mathfrak{g}}{\varkappa} .
\end{gathered}
$$

Here $Q^{\vee}$ is considered as a sublattice in $Q$ via the identification $\mathfrak{h} \simeq \mathfrak{h}^{*}$ given by $($,$) .$

Proof. This follows from the results of Kac and Peterson (see below) and the "strange formula of Freudental-de Vries" (see [Kac, 12.1.8]):

$$
\frac{\operatorname{dim} \mathfrak{g}}{24}=\frac{(\rho, \rho)}{2 h^{\vee}} .
$$

We will give an elementary proof of the identity $s^{2}=D^{2} c$, where $D$ is given by (3.19) in Section 6. The formula for $\zeta$ can be proved similarly.

Note that (3.19) implies the following formula for the renormalized $s, t$ matrices:

$$
\begin{gathered}
\tilde{s}_{\lambda \mu}=\mathrm{i}^{\left|R^{+}\right|}\left|P / \varkappa Q^{\vee}\right|^{-1 / 2} \sum_{w \in W}(-1)^{l(w)} e^{-2 \pi \mathrm{i}(w(\lambda+\rho), \mu+\rho) / \varkappa}, \\
\tilde{t}_{\lambda \mu}=e^{2 \pi \mathrm{i}\left(\frac{(\lambda, \lambda+2 \rho)}{2 \varkappa}-\frac{c}{24}\right)},
\end{gathered}
$$

where $c$ is given by (3.19).

The same formulas have appeared as the matrices of modular transformations of the characters of integrable modules over affine Lie algebra of level $k=\varkappa-h^{\vee}$ (KacPeterson formula, see [Kac, Section 13.8]). In this case the number $c$ is interpreted as the central charge of the Virasoro algebra. We will discuss the relation between affine Lie algebras and quantum groups in forthcoming papers. 
Remark 3.10. In fact, the results of this section are valid in a more general case. Namely, assume that $\varkappa$ is a rational number: $|\varkappa|=p / q, p, q \geq 1,(p, q)=1$. Then all the results above except for Theorem 3.9 are valid with appropriate changes indicated below. However, if $q$ is not relatively prime with $\left|P / Q^{\vee}\right|$, then the matrix $s$ may be singular and thus $\mathcal{C}$ is not a modular category; however, it is still a semisimple ribbon category with a finite number of simple objects.

1. Assume that $(m, q)=1$. Then the alcove $C$ must be taken to be

$$
C=\left\{\lambda \in P^{+} \mid\left\langle\lambda+\rho, \theta^{\vee}\right\rangle<p\right\}=\left\{\lambda \in P^{+} \mid(\lambda+\rho, \alpha)<p \text { for all } \alpha \in R^{+}\right\},
$$

and we must consider the action of affine Weyl group of level $p$ rather than $\varkappa$ : $\widetilde{W}=W \ltimes p Q^{\vee}$.

2. Assume that $q$ is divisible by $m$. In this case we must take

$$
C=\left\{\lambda \in P^{+} \mid\left\langle\lambda+\rho, \alpha^{\vee}\right\rangle<p \text { for all } \alpha \in R^{+}\right\}=\left\{\lambda \in P^{+} \mid\left\langle\lambda+\rho, \phi^{\vee}\right\rangle<p\right\},
$$

where $\phi \in R^{+}$is such that $\phi^{\vee}$ is the highest root of $R^{\vee}$, and the Weyl group $\widetilde{W}$ should be replaced by the Weyl group $\widetilde{W}^{\natural}=W \ltimes p Q$, which is the affine Weyl group corresponding to $R^{\vee}$. In this case the order of $\varepsilon$ is relatively prime to $m$. This case was considered in earlier papers of Andersen et al., and for prime $p$ it is related with representations of algebraic groups in characteristic $p$.

\section{Hermitian structure on $\mathcal{C}(\mathfrak{g}, \varkappa)$}

In this section we define a hermitian structure on the category $\mathcal{C}(\mathfrak{g}, \varkappa)$ in the sense of Section 1. To the best of my knowledge, these results are new; however, they are closely related with the results of Wenzl (see [We]) who considered unitarity of corresponding representations of the Hecke and Birman-Wenzl algebras.

This hermitian structure does not rely on the fact that $q$ is a root of unity. Therefore, in this section we consider the more general case: $U_{q} \mathfrak{g}$ is regarded as an algebra over $\mathbb{C}_{q}$ with the conjugation ${ }^{-}$in $\mathbb{C}_{q}$ which extends complex conjugation on $\mathbb{C}$ by $\overline{q^{a}}=q^{-a}, a \in \frac{1}{2 N} \mathbb{Z}$.

As we will show, this hermitian structure is essentially equivalent to defining an invariant hermitian form on representations of $U_{q} \mathfrak{g}$ satisfying certain conditions. To do it, we first need to define a $*$-algebra structure (that is, a certain involution) on $U_{q} \mathfrak{g}$.

Recall that the involution $\lambda \mapsto-w_{0}(\lambda)$, where $w_{0}$ is the longest element of the Weyl group, preserves the set of simple roots. Thus, we have an involution $\vee:[1, \ldots, r] \rightarrow[1, \ldots, r]$ such that $\alpha_{i \vee}=-w_{0}\left(\alpha_{i}\right)$.

Lemma 4.1. There exists a unique antilinear algebra automorphism $\omega: U_{q} \mathfrak{g} \rightarrow$ $U_{q} \mathfrak{g}$ such that

$$
\begin{aligned}
\omega: e_{i} & \mapsto e_{i^{\vee}}, \\
f_{i} & \mapsto f_{i^{\vee}}, \\
q^{h} & \mapsto q^{w_{0}(h)}, \\
q & \mapsto q^{-1} .
\end{aligned}
$$


The $\omega$ so defined is a coalgebra antiautomorphism and satisfies

$$
\begin{gathered}
\omega^{2}=1, \quad S \omega=\omega S^{-1}, \\
\omega(\mathcal{R})=\mathcal{R}^{-1},
\end{gathered}
$$

where $\omega$ is extended to $U_{q} \mathfrak{g}^{\otimes 2}$ by $\omega(a \otimes b)=\omega(a) \otimes \omega(b)$.

Proof. Let ${ }^{-}: U_{q} \mathfrak{g} \rightarrow U_{q} \mathfrak{g}$ be the antilinear involution such that $\overline{e_{i}}=e_{i}, \overline{f_{i}}=f_{i}$, and $\overline{q^{h}}=q^{-h}$ (this is slightly different from the definition of bar involution in [L5] due to a different choice of generators). One easily checks that this is a coalgebra antiautomorphism, satisfying $\overline{S x}=S^{-1} \bar{x}$ and $\overline{\mathcal{R}}=\mathcal{R}^{-1}$. Composing it with the $\left(\mathbb{C}_{q^{-}}\right.$-linear) involution $e_{i} \mapsto e_{i^{\vee}}, f_{i} \mapsto f_{i^{\vee}}, q^{h} \mapsto q^{-w_{0}(h)}$, which obviously preserves all structures of $U_{q} \mathfrak{g}$, we get $\omega$.

Now, for every module $V$ over $U_{q} \mathfrak{g}$ we define the new module $V^{\omega}$ as follows: as a set (and moreover, as an $\mathbb{R}$-vector space) $V^{\omega}$ coincides with $V$, and the action of $U_{q} \mathfrak{g}$ is defined by $\pi_{V^{\omega}}(x)=\pi(\omega x)$. It is easy to see that if $V=V_{\lambda}, \lambda \in P^{+}$, then $V^{\omega} \simeq V_{\lambda^{*}}$. For a vector $v \in V$ we will write $v^{\omega}$ to denote the same vector considered as an element of $V^{\omega}$; similarly, if $\Phi \in \operatorname{Hom}_{U_{q} \mathfrak{g}}(V, W)$, then $\Phi$ is also an intertwiner regarded as a map $V^{\omega} \rightarrow W^{\omega}$; we will denote it by $\Phi^{\omega}$.

It follows from the fact that $\omega$ is coalgebra antiautomorphism that the map $(v \otimes w)^{\omega} \mapsto w^{\omega} \otimes v^{\omega}$ is an isomorphism $(V \otimes W)^{\omega} \simeq W^{\omega} \otimes V^{\omega}$.

In particular, this implies that if $\check{R}_{V, W}: V \otimes W \rightarrow W \otimes V$ is the commutativity isomorphism defined above, then

$$
\check{R}_{V, W}^{\omega}=\left(\check{R}_{V^{\omega}, W^{\omega}}\right)^{-1}: W^{\omega} \otimes V^{\omega} \rightarrow V^{\omega} \otimes W^{\omega} .
$$

Now, since $V_{\lambda}^{\omega} \simeq V_{\lambda^{*}}$, we can identify $V_{\lambda}^{\omega} \simeq\left(V_{\lambda}\right)^{*}$. In other words, there is a unique up to a constant $U_{q} \mathfrak{g}$-homomorphism $V_{\lambda}^{\omega} \otimes V_{\lambda} \rightarrow \mathbb{C}_{q}$, or a non-degenerate hermitian form $H$ in $V_{\lambda}$ such that

$$
H\left(x v, v^{\prime}\right)=H\left(v, x^{*} v^{\prime}\right),
$$

where $x^{*}=S \omega(x)$. This form can be chosen so that it satisfies the usual symmetry condition $H(v, w)=\overline{H(w, v)}$. Note that, contrary to usual convention, this form is antilinear in the first argument and linear in the second.

As we said above, this form is defined uniquely up to a non-zero complex factor, and there is no canonical choice of this form. Note, however, that this form cannot be positive definite: if $v \in V[\lambda], v^{\prime} \in V\left[\lambda^{\prime}\right]$, then $H\left(v, v^{\prime}\right)=0$ unless $\lambda=w_{0}\left(\lambda^{\prime}\right)$; in particular, $H(v, v)=0$ unless $v \in V[0]$.

Since every module is completely reducible, we can choose an identification $V^{\omega} \simeq V^{*}$ for every $V$. Moreover, we can do it in such a way that this is compatible with tensor product and duality, i.e. the identification $(V \otimes W)^{\omega} \simeq(V \otimes W)^{*}$ coincides with the composition $(V \otimes W)^{\omega} \simeq W^{\omega} \otimes V^{\omega} \simeq W^{*} \otimes V^{*} \simeq(V \otimes W)^{*}$, and the identification $V=V^{\omega \omega} \simeq\left(V^{*}\right)^{\omega} \simeq V^{* *}$ coincides with $\delta_{V}$.

Thus, if $\Phi$ is an intertwiner $V \rightarrow W$, then $\Phi^{\omega}$ can also be considered as an intertwiner $V^{*} \rightarrow W^{*}$, which gives us the following result:

Theorem 4.2. The map $\Phi \mapsto \Phi^{\omega}$ defined above endows Rep $U_{q} \mathfrak{g}$ with a structure of hermitian category over the field $\mathbb{C}_{q}$ with respect to the above defined complex conjugation on $\mathbb{C}_{q}$. 
Proof. We have to check consistency relations (1.22). It follows from (4.2) that $\check{R}_{V, W}=\left(\check{R}_{V^{*}, W^{*}}\right)^{-1}$; the relation $\bar{\theta}=\theta^{-1}$ is obvious since $\theta$ has eigenvalues $q^{(\lambda, \lambda+2 \rho)^{\prime}}$, and the commutation relation with $e_{V}$ follows from compatibility with duality (see above) and symmetry of $H$.

Remark. This result can be slightly generalized. Namely, if we have a central element $Z$ such that $\Delta Z=Z \otimes Z, S(Z)=Z^{-1}$, and $Z^{2}=1$, then we can modify the definition of hermitian structure by choosing the isomorphism $V^{\omega \omega} \simeq V$ to be given by $v \mapsto Z v$ and replacing the symmetry condition for $H$ by $H\left(v_{2}, v_{1}\right)=\overline{H\left(Z v_{1}, v_{2}\right)}$. One easily checks that Theorem 4.2 remains valid. If we are only interested in inner products on the spaces of morphisms between tensor products of irreducibles, then up to \pm 1 these hermitian structures give the same answer.

The conjugation $\omega$ works as well if we replace $q$ by a root of unity $\varepsilon$. Moreover, it is easy to see that $\omega$ preserves Weyl (and thus, tilting) modules and that a morphism $\Phi$ is negligible iff $\Phi^{\omega}$ is negligible. Obviously, $V_{\lambda}^{\omega} \simeq V_{\lambda}^{*}$ if $\lambda \in C$; thus, the construction above defines a structure of hermitian category on $\mathcal{C}(\mathfrak{g}, \varkappa)$.

Having defined the hermitian structure, we can define inner product on intertwiners $\operatorname{Hom}_{\mathcal{C}(\mathfrak{g}, \varkappa)}(V, W)$. In fact, the construction above gives even more: it gives an inner product on a larger space $\operatorname{Hom}_{U_{\varepsilon}}(V, W)$ if $V, W$ are modules over $U_{\varepsilon}$. Recall that by definition we have

$$
\operatorname{Hom}_{\mathcal{C}(\mathfrak{g}, \varkappa)}(V, W)=\operatorname{Hom}_{U_{\varepsilon}}(V, W) / \operatorname{Hom}_{U_{\varepsilon}}^{n e g}(V, W),
$$

where $\mathrm{Hom}^{\text {neg }}$ is the space of negligible morphisms.

Lemma 4.3. Let $\lambda, \mu, \nu \in C$. Then $\Phi \in \operatorname{Hom}_{U_{\varepsilon}}\left(V_{\lambda}^{\varepsilon} \otimes V_{\mu}^{\varepsilon}, V_{\nu}^{\varepsilon}\right)$ is negligible iff $\Phi$ is in the kernel of the inner product $($, ) defined by $(2.2)$.

Proof. If $\Phi$ is negligible, then $\left(\Phi, \Phi^{\prime}\right)$ can be rewritten as a trace of some operator in a negligible module $Z$, and thus is equal to zero. Vice versa, assume that $\Phi$ lies in the kernel of this inner product. Since the inner product of intertwiners in $\mathcal{C}(\mathfrak{g}, \varkappa)$ is non-degenerate (Theorem 2.2), this shows that $\Phi=0$ as a homomorphism in $\mathcal{C}(\mathfrak{g}, \varkappa)$, and thus, by definition, $\Phi$ is negligible.

Finally, let us consider the inner product on the spaces $\operatorname{Hom}(V, V \otimes U)$. It turns out that in this case the inner product on intertwiners coincides with the inner product on so-called generalized characters (see [EK1]-[EK3]), definition of which we briefly recall below. The arguments below work for both categories $\mathcal{C}(\mathfrak{g}, \varkappa)$ (over $\mathbb{C})$ and for $\operatorname{Rep} U_{q} \mathfrak{g}$ (over $\mathbb{C}_{q}$ ); for simplicity, we will formulate all results for $\mathcal{C}(\mathfrak{g}, \varkappa)$.

For an intertwiner $\Phi \in \operatorname{Hom}_{U_{\varepsilon}}(V, V \otimes U)$ define the corresponding generalized character $\chi_{\Phi} \in \mathbb{C}[P] \otimes U[0]$ by

$$
\chi_{\Phi}=\sum_{\lambda \in P} \operatorname{Tr}_{V[\lambda]}(\Phi) .
$$

Equivalently, we can consider $\chi_{\Phi}$ as a function on $\mathfrak{h}$ by letting $e^{\lambda}(h)=e^{\langle\lambda, h\rangle}$; then the above definition is equivalent to

$$
\chi_{\Phi}(h)=\operatorname{Tr}_{V}\left(\Phi e^{h}\right) .
$$

Let us define the following involution on $\mathbb{C}[P]$ :

$$
\overline{\sum a_{\lambda} e^{\lambda}}=\sum \overline{a_{\lambda}} e^{-w_{0}(\lambda)} .
$$


Then one can define the following inner product on $\mathbb{C}[P] \otimes U[0]$ :

$$
\left(\chi_{1}, \chi_{2}\right)_{1}=\frac{(-1)^{\left|R^{+}\right|}}{|W|}\left[\left(\chi_{1} \otimes \bar{\chi}_{2}\right)_{U} \delta \bar{\delta}\right]_{0}
$$

(the subscript 1 will be explained later when we generalize this inner product introducing $\left.(,)_{k}\right)$. Here $\delta$ is Weyl denominator $(3.9),(\cdot \otimes \cdot)_{U}:(\mathbb{C}[P] \otimes U[0])^{\otimes 2} \rightarrow \mathbb{C}[P]$ is composition of the hermitian form $H: U \otimes U \rightarrow \mathbb{C}$ discussed above and multiplication in $\mathbb{C}[P]$, and

$$
\left[\sum a_{\lambda} e^{\lambda}\right]_{0}=a_{0} .
$$

Then we have the following theorem which is the hermitian analogue of a statement proved in [EK2]:

Theorem 4.4. Assume that $V$ is an irreducible representation of $U_{\varepsilon}$. Let $\Phi_{1}, \Phi_{2} \in$ $\operatorname{Hom}_{U_{\varepsilon}}(V, V \otimes U)$, and let $\chi_{1}, \chi_{2} \in \mathbb{C}[P] \otimes U[0]$ be the corresponding generalized characters. Then

$$
\left(\Phi_{1}, \Phi_{2}\right)=\frac{1}{\sqrt{\operatorname{dim}_{\varepsilon} U}}\left(\chi_{1}, \chi_{2}\right)_{1} .
$$

Proof. The proof repeats that of [EK2] with minor changes and is based on the identity $\left(\chi_{1} \otimes \bar{\chi}_{2}\right)_{U}=\chi_{\Psi}$, where the intertwiner $\Psi: V \otimes V^{*} \rightarrow V \otimes V^{*}$ is given by

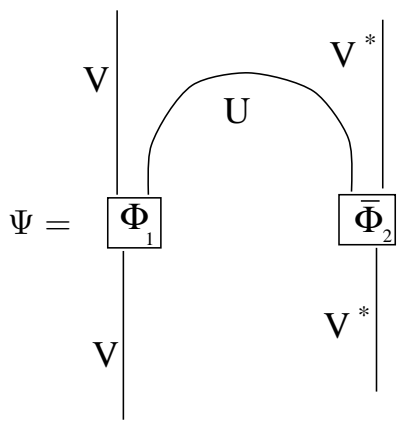

This along with the well-known identity $\left[\chi_{\lambda} \delta \bar{\delta}\right]_{0}=\delta_{\lambda, 0}(-1)^{\mid R^{+}}|W|$ proves the theorem.

\section{MACDONALD'S THEORY}

In this section we consider an example of the action of modular group in the modular tensor category obtained from quantum groups at roots of unity. Namely, we consider $\mathfrak{g}=\mathfrak{s l}_{n}$ and $U$, a symmetric power of fundamental representation. We will show that in this case the $S$-matrix can be written in terms of Macdonald's polynomials of type $A_{n-1}$, and deduce from this certain identities for values of these polynomials at roots of unity.

Even though in this case one can write explicitly $\rho, h^{\vee}$, we will use the general notations as far as possible.

As in Section 3, we consider the reduced category $\mathcal{C}\left(\mathfrak{s l}_{n}, \varkappa\right)$, based on representations of $U_{\varepsilon}$, where $\varepsilon=e^{\pi \mathrm{i} / \varkappa}$. Let us fix a positive integer $k$ and assume that $\varkappa$ has the following form:

$$
\varkappa=K+k h^{\vee}, \quad K \in \mathbb{Z}_{+} .
$$


Define

$$
C_{K}=\left\{\lambda \in P^{+} \mid\left\langle\lambda, \theta^{\vee}\right\rangle \leq K\right\} .
$$

Equivalently, we can rewrite this condition as follows: for any $\alpha \in R^{+}$,

$$
\left\langle\lambda+k \rho, \alpha^{\vee}\right\rangle<\varkappa-(k-1) .
$$

Note that $C_{K}$ is non-empty and $\lambda \in C_{K} \Longleftrightarrow \lambda^{*} \in C_{K}$.

Example. For $k=1$, this coincides with the domain $C$ we defined in Section 3.

Let $U=V_{(k-1) n \omega_{1}}$, where $\omega_{1}$ is the first fundamental weight; in other words, $U$ is the deformation of the module $S^{(k-1) n} \mathbb{C}^{n}$, where $\mathbb{C}^{n}$ is the fundamental representation of $\mathfrak{s l}_{n}$. Note that due to Lemma 3.2, $U$ is an irreducible module over $U_{\varepsilon}$. It will be extremely important for us that $U[0]$ is one-dimensional; we fix some non-zero vector $u_{0} \in U[0]$, which allows us to identify $U[0] \simeq \mathbb{C}: u_{0} \mapsto 1$.

Theorem 5.1. Let $\mu \in C$. Then

$$
\operatorname{dim} \operatorname{Hom}_{\mathcal{C}\left(\mathfrak{s l}_{n}, \varkappa\right)}\left(V_{\mu}, V_{\mu} \otimes U\right)= \begin{cases}1, & \mu=\lambda+(k-1) \rho \text { for some } \lambda \in C_{K}, \\ 0 & \text { otherwise. }\end{cases}
$$

Note that $\lambda \in C_{K}$ implies $\lambda+(k-1) \rho \in C$, so $V_{\lambda+(k-1) \rho}$ is irreducible over $U_{\varepsilon}$. We will prove this theorem later.

From now on, for simplicity let us denote

$$
\lambda^{k}=\lambda+(k-1) \rho .
$$

As before, let us denote $H=\bigoplus_{\mu \in C} V_{\mu}^{*} \otimes V_{\mu}$. Theorem 5.1 allows us to choose a basis in $\operatorname{Hom}(H, U)$. Indeed, we have canonical isomorphism $\operatorname{Hom}\left(V_{\mu}^{*} \otimes V_{\mu}, U\right) \simeq$ $\operatorname{Hom}\left(V_{\mu}, V_{\mu} \otimes U\right)$. For $\lambda \in C_{K}$, let $\Phi_{\lambda}: V_{\lambda^{k}} \rightarrow V_{\lambda^{k}} \otimes U$ be an intertwiner such that $\Phi\left(v_{\lambda^{k}}\right)=v_{\lambda^{k}} \otimes u_{0}+\ldots$. It follows from Theorem 5.1 that such an intertwiner exists and is unique and that

$$
\operatorname{Hom}(H, U) \simeq \bigoplus_{\lambda \in C} \operatorname{Hom}\left(V_{\lambda}, V_{\lambda} \otimes U\right)=\bigoplus_{\lambda \in C_{K}} \mathbb{C} \Phi_{\lambda} .
$$

The main result of this section is that in this basis the action of the matrix $S_{U}$ defined in Theorem 1.10 is given by the values of Macdonald's polynomials at special points.

Recall that Macdonald's polynomials $P_{\lambda}^{q, q^{k}}$ (where $k$ is the same positive integer that we used at the beginning of this section) are the elements of $\mathbb{C}(q)[P]^{W}$ which are defined by the following conditions (see [M1], [M2]):

1. $P_{\lambda}=e^{\lambda}+$ lower order terms.

2. $\left(P_{\lambda}, P_{\mu}\right)_{k}=0$ if $\lambda \neq \mu$, where

$$
(f, g)_{k}=\frac{(-1)^{k \mid R^{+}} \mid}{|W|}\left[f \bar{g} \delta_{k} \overline{\delta_{k}}\right]_{0} .
$$

Here

$$
\delta_{k}=\prod_{i=0}^{k-1} \prod_{\alpha \in R^{+}}\left(e^{\alpha / 2}-q^{-2 i} e^{-\alpha / 2}\right),
$$

and all other notations are as in Section 4 with complex conjugation in $\mathbb{C}$ extended to $\mathbb{C}(q)$ by $\bar{q}=q^{-1}$. 
Remark. This definition, as well as the complex conjugation on $\mathbb{C}(q)[P]$, differs from the definition in both original Macdonald's papers and [EK1]-[EK3], which use the $\mathbb{C}(q)$-linear inner product rather than hermitian. However, it is easy to check that this definition is in fact equivalent to the original one, which relies on the identity

$$
P_{\lambda}^{q^{-1}, q^{-k}}=P_{\lambda}^{q, q^{k}}
$$

(see [M1]).

We use the same notations as we did in [EK2]; thus, what we denote by $P_{\lambda}^{q, q^{k}}$, in the original notations of Macdonald would be $P_{\lambda}\left(x ; q^{2}, q^{2 k}\right)$.

From now on, we will drop the superscript $q, q^{k}$ and denote Macdonald's polynomials simply $P_{\lambda}$. The following properties of these polynomials can be easily deduced from the definition:

$$
\begin{aligned}
\overline{P_{\lambda}} & =P_{\lambda^{*}}, \\
\overline{P_{\lambda}\left(q^{\mu}\right)}=P_{\lambda^{*}}\left(q^{\mu}\right) & =P_{\lambda}\left(q^{-\mu}\right)=P_{\lambda}\left(q^{\mu^{*}}\right) .
\end{aligned}
$$

Here ${ }^{-}$is the involution in $\mathbb{C}_{q}$ (in the second line) and in $\mathbb{C}_{q}[P]$ (in the first line) which was defined in Section 4.

Our arguments will be based on the relation between Macdonald's polynomials of type $A$ and representations of $U_{q} \mathfrak{s l}_{n}$. We recall the main facts here, following the papers [EK2], [EK3]; note, however, that the quantum group used in these papers differs from the one used here by substitution $q \leftrightarrow q^{-1}$.

For the moment, we consider representations of $U_{q} \mathfrak{s l}_{n}$ for a generic $q$, i.e. over the field $\mathbb{C}_{q}=\mathbb{C}\left(q^{1 / 2 n}\right)$. Let $k, U, u_{0}$ be the same as above. Then we have the following results (see [EK2], [EK3]):

1.

$$
\operatorname{dim} \operatorname{Hom}_{U_{q} \mathfrak{s l}_{n}}\left(V_{\mu}, V_{\mu} \otimes U\right)= \begin{cases}1, & \mu=\lambda+(k-1) \rho \text { for some } \lambda \in P_{+}, \\ 0 & \text { otherwise. }\end{cases}
$$

We fix an intertwiner $\Phi_{\lambda}: V_{\lambda^{k}} \rightarrow V_{\lambda^{k}} \otimes U$ such that $\Phi_{\lambda} v_{\lambda^{k}}=v_{\lambda^{k}} \otimes u_{0}+\ldots$

2. Let $\varphi_{\lambda} \in \mathbb{C}(q)[P] \otimes U[0]$ be the generalized character of $\Phi_{\lambda}$ (see (3.14)). Then

$$
P_{\lambda}=\frac{\varphi_{\lambda}}{\varphi_{0}}
$$

$$
\varphi_{0}=\prod_{\alpha \in R^{+}} \prod_{i=1}^{k-1}\left(e^{\alpha / 2}-q^{-2 i} e^{-\alpha / 2}\right) \cdot u_{0}
$$

3.

$$
\left(\Phi_{\lambda}, \Phi_{\lambda}\right)=\frac{(-1)^{(k-1)\left|R^{+}\right|}}{\sqrt{\operatorname{dim}_{q} U}}\left(u_{0}, u_{0}\right)\left(P_{\lambda}, P_{\lambda}\right)_{k}
$$

where

$$
\left(P_{\lambda}, P_{\lambda}\right)_{k}=\prod_{\alpha \in R^{+}} \prod_{i=1}^{k-1} \frac{[(\alpha, \lambda+k \rho)+i]}{[(\alpha, \lambda+k \rho)-i]} .
$$

This identity is a reformulation of famous Macdonald's inner product identities in our case, i.e. for hermitian rather than bilinear inner product.

Now we can come back to Theorem 5.1. 
Proof of Theorem 5.1. It follows from Lemma 3.7 that $\operatorname{dim} \operatorname{Hom}_{\mathcal{C}}\left(V_{\mu}, V_{\mu} \otimes U\right) \leq 1$, and it can be non-zero only if $\mu=\lambda+(k-1) \rho, \lambda \in P^{+}$. It follows from Lemma 4.3 that this dimension is equal to one iff $\left(\Phi_{\lambda}, \Phi_{\lambda}\right) \neq 0$, where $\Phi_{\lambda}$ is the corresponding intertwiner for $U_{\varepsilon}$. On the other hand, formula (5.7) shows that $\left(\Phi_{\lambda}, \Phi_{\lambda}\right) \neq 0 \Longleftrightarrow$ $\lambda \in C_{K}$.

Note that the proof used a highly non-trivial result - an explicit formula for the norm $\left(\Phi_{\lambda}, \Phi_{\lambda}\right)$ (Macdonald's inner product formula).

Now, let us come back to the case of roots of unity. Let $\lambda \in C_{K}$, and let $\Phi_{\lambda} \in \operatorname{Hom}_{\mathcal{C}(\mathfrak{g}, \varkappa)}\left(V_{\lambda^{k}}^{*} \otimes V_{\lambda^{k}}, U\right)$ be as before.

Theorem 5.2. Let $\lambda \in C_{K}$. Then $P_{\lambda}$ is well defined at $q=\varepsilon$ (i.e., its coefficients, which are rational functions of $q$, are well-defined at $q=\varepsilon$ ).

Proof. This follows from the fact that Macdonald's polynomials can be written in terms of generalized characters (see (5.6)) and Lemma 3.7.

Now comes the crucial step.

Lemma 5.3. We have the following identity in the category $\mathcal{C}\left(\mathfrak{s l}_{n}, \varkappa\right)$ :

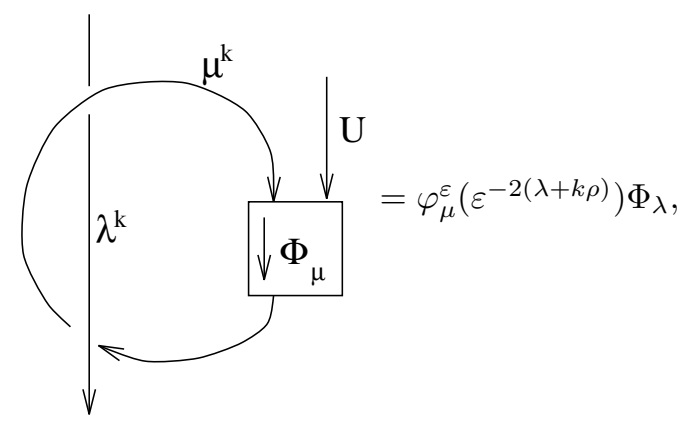

where $\varphi_{\lambda}^{\varepsilon}$ is the element of $\mathbb{C}[P]$ which is obtained by substituting $q=\varepsilon$ in the expression for $\varphi_{\lambda}$ and identifying $U[0] \simeq \mathbb{C}: u_{0} \mapsto 1$.

Proof. For the case when $q$ is indeterminate, it was proved in [EK3]; it is easy to see that in fact all the arguments can be carried out in the case $q=\varepsilon$ as well.

This immediately implies the following theorem:

Theorem 5.4. Let $\mathfrak{g}=\mathfrak{s l}_{n}$, and let $U, \Phi_{\lambda}, \lambda \in C_{K}$ be as above. Then the action of the modular group in $\operatorname{Hom}(H, U)$ in this basis is given by the matrices $S_{U}=$ $\left(S_{\lambda \mu}\right), T_{U}=\left(T_{\lambda \mu}\right)$, where

$$
\begin{gathered}
T_{\lambda \mu}=\delta_{\lambda \mu} \varepsilon^{(\lambda+k \rho, \lambda+k \rho)-\frac{\varkappa}{n}(\rho, \rho)} \\
S_{\lambda \mu}=d_{\lambda} P_{\mu}^{\varepsilon}\left(\varepsilon^{-2(\lambda+k \rho)}\right)
\end{gathered}
$$

where $P_{\lambda}^{\varepsilon}$ is Macdonald's polynomial $P_{\lambda}^{q, q^{k}}$ calculated at $q=\varepsilon$, and

$$
d_{\lambda}=\frac{i^{n(n-1) / 2}}{\sqrt{n} \varkappa^{(n-1) / 2}} \prod_{\alpha \in R^{+}} \prod_{i=0}^{k-1}\left(\varepsilon^{-(\alpha, \lambda+k \rho)}-\varepsilon^{-2 i+(\alpha, \lambda+k \rho)}\right) .
$$


Proof. Formula for $T$ is obvious from formula (3.19) for $\zeta$ and $\left(\lambda^{k}, \lambda^{k}+2 \rho\right)=$ $(\lambda+k \rho, \lambda+k \rho)-(\rho, \rho)$. It follows from the definition of $S$ and Lemma 5.3 that $S_{\lambda \mu}$ is given by formula (5.8) with

$$
d_{\lambda}=\frac{\operatorname{dim}_{\varepsilon} V_{\lambda^{k}}}{D} \varphi_{0}\left(\varepsilon^{-2(\lambda+k \rho)}\right)
$$

(as before, we consider $\varphi_{0}$ as scalar-valued). Substituting in this expression the Weyl formula for $\operatorname{dim}_{\varepsilon} V_{\lambda^{k}}$, expression (3.19) for $D$ and formula (5.6) for $\varphi_{0}$, we see that

$$
d_{\lambda}=\frac{\mathrm{i}^{\left|R^{+}\right|}}{\sqrt{|P| \varkappa Q \mid}} \prod_{\alpha \in R^{+}} \prod_{i=0}^{k-1}\left(\varepsilon^{-(\alpha, \lambda+k \rho)}-\varepsilon^{-2 i+(\alpha, \lambda+k \rho)}\right) .
$$

Since for $\mathfrak{s l}_{n}$ we have $|P / Q|=n,\left|R^{+}\right|=n(n-1) / 2$, and the rank is $n-1$, we get formula (5.9).

Similar formulas for the action of $S L_{2}(\mathbb{Z})$ in terms of the values of Macdonald's polynomials were obtained by Cherednik $([\mathrm{Ch}])$ in the study of difference Fourier transform.

Example. Consider the case $\mathfrak{g}=\mathfrak{s l}_{2}$. Then every irreducible finite-dimensional representation has the form $V_{(k-1) n \omega_{1}}$ for some choice of $k$, and thus, in this case Theorem 5.4 gives all matrix coefficients of the action of the modular group in $H$, which in this case are written in terms of $q$-ultraspherical polynomials (=Macdonald's polynomials for $\mathfrak{s l}_{2}$ ). In particular, this shows that the $S$-matrix can be written in terms of basic hypergeometric functions with parameter $q$ taken to be a root of unity (see [AI] for expressions of $q$-ultraspherical polynomials in terms of basic hypergeometric functions).

Explicit calculation, using symmetry properties (5.5) of Macdonald's polynomials, gives the following symmetries of the $S$-matrix:

$$
\begin{gathered}
S_{\lambda \mu}=S_{\lambda^{*} \mu^{*}}, \\
\overline{S_{\lambda \mu}}=(-1)^{(k-1) n(n-1) / 2} \varepsilon^{n(n-1) k(k-1) / 2} S_{\lambda^{*} \mu} .
\end{gathered}
$$

Also, it is easy to calculate the action of the matrix $C$ in the basis $\Phi_{\lambda}$. As before, let us assume that we have chosen identifications $V_{\lambda^{*}} \simeq V_{\lambda}^{*}$ as in (1.1). Then we have the following theorem:

\section{Theorem 5.5.}

$$
\Phi_{\lambda} C^{-1}=(-1)^{(k-1) n(n-1) / 2} \varepsilon^{n(n-1) k(k-1) / 2} \Phi_{\lambda^{*}} .
$$

Proof. Let $v_{\lambda^{k}}$ be the highest-weight vector in $V_{\lambda^{k}}$, and $v_{\lambda^{k}}^{*}$ the lowest weight vector in $V_{\lambda^{k}}^{*}$ such that $\left\langle v_{\lambda^{k}}^{*}, v_{\lambda^{k}}\right\rangle=1$. Also, let $w_{\lambda^{k}}$ be the lowest weight vector in $V_{\lambda^{k}}$, and $w_{\lambda^{k}}^{*}$ the highest weight vector in $V_{\lambda^{k}}^{*}$ such that $\left\langle w_{\lambda^{k}}^{*}, w_{\lambda^{k}}\right\rangle=1$. Then by definition $\Phi_{\lambda}\left(v_{\lambda^{k}}^{*} \otimes v_{\lambda^{k}}\right)=u_{0}$, and it follows from (5.6) and symmetry of Macdonald's polynomials that

$$
\Phi_{\lambda}\left(w_{\lambda^{k}}^{*} \otimes w_{\lambda^{k}}\right)=u_{0}(-1)^{(k-1) n(n-1) / 2} \varepsilon^{-n(n-1) k(k-1) / 2} .
$$


It follows from formula (3.6) for universal $R$-matrix that

$$
\begin{aligned}
\Phi_{\lambda} C^{-1}: V_{\lambda} \otimes V_{\lambda}^{*} & \rightarrow U, \\
v_{\lambda^{k}} \otimes v_{\lambda^{k}}^{*} & \mapsto \theta_{\lambda^{k}} \varepsilon^{-\left(\lambda^{k}, \lambda^{k}\right)} \Phi_{\lambda}\left(v_{\lambda^{k}}^{*} \otimes v_{\lambda^{k}}\right)=\varepsilon^{\left(\lambda^{k}, 2 \rho\right)} u_{0} .
\end{aligned}
$$

On the other hand, if we identify $V_{\lambda} \simeq\left(V_{\lambda^{*}}\right)^{*}, V_{\lambda}^{*} \simeq V_{\lambda^{*}}$ and denote by $\langle$,$\rangle the$ canonical pairing $\left(V_{\lambda^{*}}\right)^{*} \otimes V_{\lambda^{*}} \rightarrow \mathbb{C}$, then

$$
\left\langle v_{\lambda^{k}}, v_{\lambda^{k}}^{*}\right\rangle=\varepsilon^{\left(\lambda^{k}, 2 \rho\right)} .
$$

Therefore, similarly to what we discussed before,

$$
\Phi_{\lambda^{*}}\left(v_{\lambda^{k}} \otimes v_{\lambda^{k}}^{*}\right)=(-1)^{(k-1) n(n-1) / 2} \varepsilon^{-n(n-1) k(k-1) / 2} \varepsilon^{\left(\lambda^{k}, 2 \rho\right)} u_{0} .
$$

Comparing these expressions, we get the statement of the theorem.

Remark. Note that since $\theta_{U}=\varepsilon^{n(n-1) k(k-1)}$, which is verified by direct computation, we again see that $C^{2}=\theta_{U}^{-1}$.

Now we can rewrite the results about the action of modular group which were proved in a purely abstract setting in Sections 1 and 2 to this case, which results in identities for Macdonald's polynomials:

Theorem 5.6. For $\lambda, \mu \in C_{K}$,

$$
S_{\lambda \mu}\left(P_{\lambda}, P_{\lambda}\right)_{k}=S_{\mu \lambda}\left(P_{\mu}, P_{\mu}\right)_{k} .
$$

Proof. This is nothing but the condition of unitarity of the matrix $S$ with respect to the inner product on intertwiners (Theorem 2.5). Indeed, the unitarity condition can be rewritten as follows: $\left(\Phi_{\mu} S, \Phi_{\lambda}\right)=\left(\Phi_{\mu}, \Phi_{\lambda} C^{-1} S\right)$, which is equivalent to

$$
S_{\lambda \mu}\left(\Phi_{\lambda}, \Phi_{\lambda}\right)=(-1)^{(k-1) n(n-1) / 2} \varepsilon^{-n(n-1) k(k-1) / 2} \overline{S_{\mu \lambda^{*}}}\left(\Phi_{\mu}, \Phi_{\mu}\right) .
$$

Using symmetry properties (5.10), we get the statement of the theorem.

Using expressions for the $S$-matrix given in Theorem 5.4, we can rewrite this result as follows:

$$
P_{\lambda}^{\varepsilon}\left(\varepsilon^{-2(\mu+k \rho)}\right)\left(P_{\mu}, P_{\mu}\right)_{k} d_{\mu}=P_{\mu}^{\varepsilon}\left(\varepsilon^{-2(\lambda+k \rho)}\right)\left(P_{\lambda}, P_{\lambda}\right)_{k} d_{\lambda},
$$

which is precisely the symmetry identity for Macdonald's polynomials of type $A$ (see [EK3]). One can check that in fact all our arguments work for general $q$, i.e. one can avoid using the fact that the category is modular; essentially, this is the same proof that was given in [EK3], only now it has a clear interpretation.

Other identities, which only apply to modular categories and thus do not generalize to the case of indeterminate $q$ can be obtained from the relations in the modular group. This gives the following purely combinatorial theorem:

Theorem 5.7. Let $S=\left(S_{\lambda \mu}\right), T=\left(T_{\lambda \mu}\right), \lambda, \mu \in C_{K}$ be the matrices given by (5.8), (5.9). Then

$$
\begin{gathered}
S^{2}=(-1)^{(k-1) n(n-1) / 2} \varepsilon^{-n(n-1) k(k-1) / 2} \delta_{\lambda \mu^{*}}, \\
(S T)^{3}=S^{2} .
\end{gathered}
$$


These are certain identities for Macdonald's polynomials at roots of unity, which were not known before and which would be very difficult to prove by combinatorial methods. Again, similar (and even more general) identities have been recently obtained by Cherednik ([Ch]) in the study of difference Fourier transform related with double affine Hecke algebras.

\section{Characters and the Grothendieck Ring}

In this section we again return to the consideration of the category $\mathcal{C}(\mathfrak{g}, \varkappa)$ for arbitrary $\mathfrak{g}$ and describe its Grothendieck ring. We also give an elementary proof of the fact that the matrix $s_{\lambda \mu}$ defined by (3.18) is non-singular, and calculate its square. Results of this section are not new, but I was unable to locate them in the literature ${ }^{1}$ so for the sake of completeness they are included here.

Recall (see Section 3) that we have fixed $\varkappa \in \mathbb{Z}_{+}$such that $\varkappa \geq h^{\vee}$, and we have defined the open and closed alcoves

$$
\begin{gathered}
C=\left\{\lambda \in P^{+} \mid\left\langle\lambda+\rho, \theta^{\vee}\right\rangle<\varkappa\right\}, \\
\bar{C}=\left\{\lambda \in P \mid\left\langle\lambda+\rho, \alpha_{i}^{\vee}\right\rangle \geq 0,\left\langle\lambda+\rho, \theta^{\vee}\right\rangle \leq \varkappa\right\} .
\end{gathered}
$$

We have also defined the affine Weyl group $\widetilde{W}=W \ltimes \varkappa Q^{\vee}$ and its shifted action on $\mathfrak{h}^{*}$ by

$$
w \cdot \lambda=w(\lambda+\rho)-\rho .
$$

Then, as is well-known, we have the following statements:

1. $\bar{C}$ is the fundamental domain for the shifted action of $\widetilde{W}$.

2. Every $\lambda \in C$ is regular with respect to the shifted action of $\widetilde{W}: w \cdot \lambda=\lambda$ iff $w=1$.

3. For every $f \in \mathbb{C}[P]^{W}, \mu \in P, w \in \widetilde{W}$ we have

$$
f\left(\varepsilon^{2 w(\mu)}\right)=f\left(\varepsilon^{2 \mu}\right) .
$$

It fact, the last statement can be reversed: if $\lambda, \mu \in P$ are such that $f\left(\varepsilon^{2 \lambda}\right)=f\left(\varepsilon^{2 \mu}\right)$ for all $f \in \mathbb{C}[P]^{W}$, then $\lambda=w(\mu)$ for some $w \in \widetilde{W}$; however, we won't use this result.

For every $\lambda \in P$ define $\chi_{\lambda} \in \mathbb{C}[P]^{W}$ by

$$
\chi_{\lambda}=\frac{\sum_{W}(-1)^{l(w)} e^{w(\lambda+\rho)}}{\delta},
$$

where $\delta$ is Weyl denominator (3.9). For $\lambda \in P^{+}, \chi_{\lambda}$ is the character of the module $V_{\lambda}$.

Now let $\varepsilon=e^{\pi \mathrm{i} / m \varkappa}$. Recall that we denote

$$
\operatorname{dim}_{\varepsilon} V_{\lambda}=\operatorname{Tr}_{V_{\lambda}}\left(\varepsilon^{2 \rho}\right)=\chi_{\lambda}\left(\varepsilon^{2 \rho}\right)=\chi_{\lambda}\left(\varepsilon^{-2 \rho}\right) .
$$

It is easy to see that $\operatorname{dim}_{\varepsilon} V_{\lambda}=0$ for $\lambda \in \bar{C} \backslash C$, and $\operatorname{dim}_{\varepsilon} V_{\lambda} \neq 0$ for $\lambda \in C$.

\footnotetext{
${ }^{1}$ For example, it is mentioned as unknown for exceptional Lie algebras in [Tu, XI.6.4].
} 
For $\lambda, \mu \in P$, define the numbers $s_{\lambda \mu} \in \mathbb{C}$ by

$$
s_{\lambda \mu}=\frac{\sum_{w \in W}(-1)^{l(w)} \varepsilon^{-2(w(\lambda+\rho), \mu+\rho)^{\prime}}}{\delta\left(\varepsilon^{-2 \rho}\right)} .
$$

If $\lambda, \mu \in C$, this can also be rewritten as follows:

$$
s_{\lambda \mu}=\chi_{\lambda}\left(\varepsilon^{-2(\mu+\rho)}\right) \operatorname{dim}_{\varepsilon} V_{\mu} .
$$

Lemma 6.1.

$$
\begin{gathered}
s_{\lambda \mu}=s_{\mu \lambda}, \\
s_{\lambda \mu}=(-1)^{l(w)} s_{\lambda w . \mu} \quad \text { for any } w \in \widetilde{W}, \\
s_{\lambda \mu}=0 \quad \text { if } \lambda \in \bar{C} \backslash C .
\end{gathered}
$$

Theorem 6.2. Let $s=\left(s_{\lambda \mu}\right)_{\lambda, \mu \in C}$. Then

$$
s^{2}=D^{2} c,
$$

where $c_{\lambda \nu}=\delta_{\lambda \nu^{*}}$ and

$$
\begin{aligned}
D^{2} & =\left|P / \varkappa Q^{\vee}\right| \prod_{\alpha \in R^{+}}\left(2 \sin \frac{(\alpha, \rho)}{\varkappa} \pi\right)^{-2} \\
& =\left|P / \varkappa Q^{\vee}\right|(-1)^{\left|R^{+}\right|} \delta^{-2}\left(\varepsilon^{-2 \rho}\right) .
\end{aligned}
$$

Proof. Let $\lambda, \mu \in C$. Then

$$
\begin{aligned}
\sum_{\mu \in C} s_{\lambda \mu} s_{\mu \nu} & =\sum_{\mu \in \bar{C}} s_{\lambda \mu} s_{\mu \nu}=\sum_{\mu \in P / \widetilde{W}} s_{\lambda \mu} s_{\mu \nu} \\
& =\frac{1}{|W|} \sum_{\mu \in P / \varkappa Q^{\vee}} s_{\lambda \mu} s_{\mu \nu} \\
& =\frac{1}{|W| \delta^{2}\left(\varepsilon^{-2 \rho)}\right.} \sum_{\mu \in P / \varkappa Q^{\vee}} \sum_{w, w^{\prime} \in W}(-1)^{l\left(w w^{\prime}\right)} \varepsilon^{-2\left(\mu+\rho, w(\lambda+\rho)+w^{\prime}(\nu+\rho)\right)^{\prime}} .
\end{aligned}
$$

For any $a \in P$ we have

$$
\sum_{\mu \in P / \varkappa Q^{\vee}} \varepsilon^{2(\mu+\rho, a)^{\prime}}=\left\{\begin{array}{l}
0, \quad a \notin \varkappa Q^{\vee}, \\
\left|P / \varkappa Q^{\vee}\right|, \quad a \in \varkappa Q^{\vee} .
\end{array}\right.
$$

Since $\lambda, \nu \in C$, it follows from the fact that $C$ is the fundamental domain for the action of $\widetilde{W}$ that $w(\lambda+\rho)+w^{\prime}(\nu+\rho) \in \varkappa Q^{\vee}$ is only possible if $\lambda=\nu^{*}, w w^{\prime}=w_{0}$, the longest element in $W$. Thus,

$$
\sum_{\mu \in C} s_{\lambda \mu} s_{\mu \nu}=\delta_{\lambda \nu^{*}}\left|P / \varkappa Q^{\vee}\right|(-1)^{\left|R^{+}\right|} \delta^{-2}\left(\varepsilon^{-2 \rho}\right) .
$$

Corollary 6.3. The matrix $s$ defined by (6.2) is non-singular. 
In a similar way, one can prove the identity $D \zeta^{3} t^{-1} s t^{-1}=s t s$, where $D$ is as above and $\zeta$ is given by (3.19); this requires calculation of $\sum_{\mu} \varepsilon^{2(\mu, \mu+a)}$.

Now denote by $K$ the Grothendieck ring of the category $\mathcal{C}(\mathfrak{g}, \varkappa)$, and by $K_{\mathbb{C}}=$ $K \otimes_{\mathbb{Z}} \mathbb{C}$ its complexification.

Let $F(C)$ be the ring of all complex-valued functions on $C$. For every $V \in \operatorname{Rep} U_{\varepsilon}$ denote by $f_{V} \in F(C)$ the function given by

$$
f_{V}(\mu)=\operatorname{ch} V\left(\varepsilon^{-2(\mu+\rho)}\right) .
$$

Lemma 6.4. If $V$ is negligible, then $f_{V}=0$ on $C$.

Proof. It follows from the following identity:

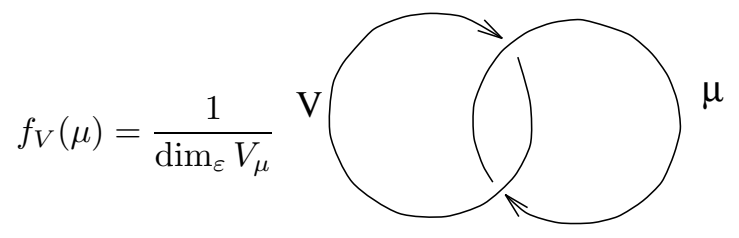

Corollary. The map $V \mapsto f_{V}$ is a well-defined ring homomorphism $K \rightarrow F(C)$.

Theorem 6.5. The map $V \mapsto f_{V}$ is an isomorphism $K_{\mathbb{C}} \simeq F(C)$.

Proof. It suffices to prove that

$$
\operatorname{det}\left(\chi_{\lambda}\left(\varepsilon^{-2(\lambda+\rho)}\right)\right)_{\lambda, \mu \in C} \neq 0,
$$

which follows from non-singularity of the matrix $s$ (Corollary 6.3).

\section{Corollary 6.6.}

$$
K_{\mathbb{C}} \simeq \mathbb{C}[P]^{W} / \mathcal{I}
$$

where the ideal $\mathcal{I}$ is spanned as a vector space by the elements of the form $\chi_{\lambda}+$ $\chi_{s_{\Gamma} \lambda}, \lambda \in P$, where $s_{\Gamma}$ is the reflection with respect to the affine wall $\Gamma$ (see (3.15)).

Remark. It can be shown (see $[\mathrm{F}]$ ) that for $\varkappa$ large enough there is a stronger result: $\mathcal{I}$ is generated as an ideal by $\chi_{\lambda}, \lambda \in \Gamma \cap P^{+}$. In particular, this is always so for $\mathfrak{g}=\mathfrak{s l}_{n}$.

\section{More on hermitian StRUCTURe in $\mathcal{C}(\mathfrak{g}, \varkappa)$}

In this section we give another description of the hermitian structure in $\mathcal{C}(\mathfrak{g}, \varkappa)$. It will be used in the future papers to establish relation with affine Lie algebras. Also, it allows us to define the inner product on the spaces of intertwiners uniquely up to a constant from $\mathbb{R}_{+}$(not from $\mathbb{C}^{\times}$, as we did in Section 4 ); thus, it makes sense to discuss whether this inner product is positive definite.

Recall that the key ingredient of the definition of hermitian structure was definition of an involution $\omega$ on $U_{q} \mathfrak{g}$, which allowed us to define for every module $V$ a module $V^{\omega}$, and isomorphisms $V^{\omega} \simeq V^{*}$.

Here is another description of the same involution. As before, we begin with consideration of generic $q$, i.e. of the quantum group over the field $\mathbb{C}_{q}$. 
Let the compact involution $\omega_{c}$ be the antilinear algebra automorphism $U_{q} \mathfrak{g} \rightarrow$ $U_{q} \mathfrak{g}$ defined by

$$
\begin{aligned}
\omega_{c}: e_{i} & \mapsto-q^{-d_{i}} f_{i}, \\
f_{i} & \mapsto-q^{d_{i}} e_{i}, \\
q^{h} & \mapsto q^{h}, \\
q & \mapsto q^{-1} .
\end{aligned}
$$

One easily checks that $\omega_{c}$ is also a coalgebra automorphism and $\omega_{c} S=S \omega_{c}$, $\omega_{c}(R)=R^{-1}, \omega_{c}^{2}=S^{2}$.

Similarly to the constructions of Section 4 , for every $U_{q} \mathfrak{g}$-module $V$ and homomorphism $\Phi$ we can define $V^{\omega_{c}}$ and $\Phi^{\omega_{c}}$. Again, for an irreducible highest-weight module $V_{\lambda}$ we have a (non-canonical) isomorphism $V_{\lambda}^{\omega_{c}} \simeq V_{\lambda}^{*}$; due to complete reducibility, the same is true for an arbitrary module $V$.

However, to use this involution to define a hermitian structure we must define isomorphisms $\left(V^{*}\right)^{\omega_{c}} \simeq\left(V^{\omega_{c}}\right)^{*},(V \otimes W)^{\omega_{c}} \simeq W^{\omega_{c}} \otimes V^{\omega_{c}}$ satisfying compatibility conditions (1.22).

The isomorphism $\left(V^{*}\right)^{\omega_{c}} \simeq\left(V^{\omega_{c}}\right)^{*}$ is trivial: as vector spaces both coincide with $V^{*}$, and this identification is an isomorphism of representations, which follows from the fact that $\omega_{c}$ commutes with $S$. As for the second isomorphism, it is less obvious: since $\omega_{c}$ is an automorphism (and not an antiautomorphism) of coalgebra structure, the map $(v \otimes w)^{\omega_{c}} \mapsto w^{\omega_{c}} \otimes v^{\omega_{c}}$ is not a morphism of representations. Instead, define the isomorphism by

$$
(v \otimes w)^{\omega_{c}} \mapsto R_{V^{\omega_{c}} W^{\omega_{c}}}\left(v^{\omega_{c}} \otimes w^{\omega_{c}}\right) .
$$

Finally, let us normalize the identification $V_{\lambda}^{\omega_{c}} \simeq V_{\lambda}^{*}$. Such an identification is equivalent to defining a pairing $V_{\lambda}^{\omega_{c}} \otimes V_{\lambda} \rightarrow \mathbb{C}$ or, equivalently, a non-degenerate hermitian form $(,)_{c}$ on $V_{\lambda}$ satisfying

$$
\left(x v, v^{\prime}\right)_{c}=\left(v, S \omega_{c}^{-1}(x) v^{\prime}\right)_{c}
$$

As before, this form is antilinear in the first argument and linear in the second.

Such a form is unique up to a constant. Let us fix this constant by the condition

$$
\left(v_{\lambda}, v_{\lambda}\right)_{c}=q^{(\lambda, \lambda+2 \rho)^{\prime} / 2}
$$

then

$$
\left(v_{1}, v_{2}\right)_{c}=\overline{\left(\theta v_{2}, v_{1}\right)_{c}}
$$

Now we can define the identification $V^{\omega_{c}} \simeq V^{*}$ for all $V$ so that this agrees with tensor products and duality.

Theorem 7.1. The so-defined functor ${ }^{*}$ defines a structure of a hermitian modular category on $\mathcal{C}(\mathfrak{g}, \varkappa)$.

It turns out that this structure is equivalent to the one defined in Section 4. The proof is based on the use of the longest element of the quantum Weyl group, which was studied in [KR], [LS], [L7]. We reformulate the results of these papers in the following theorem:

Theorem 7.2. There exists an element $\Omega$ in a certain completion of $U_{q} \mathfrak{g}$ satisfying the following properties:

1. $\Omega$ acts in every finite-dimensional $U_{q} \mathfrak{g}$-module, and $\Omega V[\lambda] \subset V\left[w_{0}(\lambda)\right]$. 
2.

$$
\begin{gathered}
\Omega f_{i} \Omega^{-1}=-q^{d_{i}} e_{i^{\vee}}, \\
\Omega e_{i} \Omega^{-1}=-q^{-d_{i}} f_{i^{\vee}}, \\
\Omega q^{h} \Omega^{-1}=q^{w_{0}(h)} .
\end{gathered}
$$

3.

$$
\Delta(\Omega)=\mathcal{R}^{-1}(\Omega \otimes \Omega) .
$$

4. $\Omega^{2}=Z \theta^{-1}$, where $\theta$ is the universal twist shown in Figure $1 a$ (recall that $\theta$ is a central element such that $\left.\left.\theta\right|_{V_{\lambda}}=q^{(\lambda, \lambda+2 \rho)^{\prime}} \mathrm{Id}\right)$, and $Z$ is a central element satisfying $\Delta Z=Z \otimes Z$ and $Z^{2}=1$.

This definition differs from the one in $[\mathrm{KR}]$ by $\Omega \rightarrow \Omega \cdot q^{2 \rho}$.

Remark 7.3. It is convenient to think of $\Omega$ as represented by the following ribbon graph:

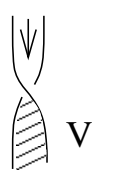

$\Omega$

Such a graph is not allowed in the original formalism developed by Reshetikhin and Turaev (and indeed, $\Omega$ is not an intertwining operator); however, this becomes possible after suitable extension of the formalism.

Comparing (7.5) with the definition of $\omega$ in Lemma 4.1 we see that

$$
\omega(x)=\Omega \cdot \omega_{c}(x) \Omega^{-1} .
$$

Now we can come back to defining the isomorphisms $V^{\omega} \simeq V^{*}$. Such an isomorphism is equivalent to defining a pairing $V^{\omega} \otimes V \rightarrow \mathbb{C}_{q}$, or a non-degenerate hermitian form on $V$ such that

$$
H\left(x v, v^{\prime}\right)=H\left(v, S \omega(x) v^{\prime}\right)
$$

Similarly, recall that we have defined a non-degenerate hermitian form $(,)_{c}$ on $V$ satisfying

$$
\left(x v, v^{\prime}\right)_{c}=\left(v, S \omega_{c}^{-1}(x) v^{\prime}\right)_{c}
$$

In fact, given any of these forms we can define the other:

Lemma 7.4. If a hermitian form $(,)_{c}$ on $V$ satisfies condition (7.3), then the form

$$
H\left(v, v^{\prime}\right)=\left(\Omega^{-1} v, v^{\prime}\right)_{c}
$$

satisfies condition (7.7), and vice versa. Also, $H\left(v, v^{\prime}\right)=\overline{H\left(Z v^{\prime}, v\right)}$ iff ( , $)_{c}$ satisfies $\left(v, v^{\prime}\right)_{c}=\overline{\left(\theta v^{\prime}, v\right)_{c}}$, where $Z$ is as in Theorem 7.2.

Proof. The proof is obvious from (7.6) and $S \omega_{c}^{-1}(\Omega)=\Omega^{-1}$, which can be proved using the construction of $\Omega$ and explicit calculation for $\mathfrak{s l}_{2}$.

Recall (see remark after Theorem 4.2) that for every central element $Z$ with some additional properties we can define a structure of hermitian category on $\mathcal{C}(\mathfrak{g}, \varkappa)$. In particular, we can take $Z$ to be as in Theorem 7.2. 
Theorem 7.5. The system of maps

$$
\begin{aligned}
\Omega^{-1}: V^{\omega} & \rightarrow V^{\omega_{c}} \\
v^{\omega} & \mapsto\left(\Omega^{-1} v\right)^{\omega_{c}}
\end{aligned}
$$

is an isomorphism of functors $\omega$ and $\omega_{c}$. This isomorphism gives rise to equivalence of hermitian structures on $\mathcal{C}(\mathfrak{g}, \varkappa)$ defined in Section 4 (with $Z$ chosen as in Theorem 7.2) and the one defined in Theorem 7.1: in particular, the inner products on spaces of homomorphisms defined by these hermitian structures as in (2.2) coincide.

Note that the condition $\left(v_{\lambda}, v_{\lambda}\right)_{c}=q^{(\lambda, \lambda+2 \rho)^{\prime} / 2}$ defines the form $(,)_{c}$ on $V_{\lambda}$ uniquely up to a constant from $\mathbb{R}_{>0}$ (which is due to ambiguity in the choice of the highest-weight vector), and thus gives us a way to define the form $H$ uniquely up to a constant from $\mathbb{R}_{>0}$; before, we could only define it up to a constant from $\mathbb{R}$ by the symmetry condition. Therefore, we can define the inner product on every space of intertwiners $\operatorname{Hom}_{U_{\varepsilon}}\left(V_{\lambda}, V_{\mu} \otimes V_{\nu}\right)$ uniquely up to a real positive factor.

So far, all the results are valid both for generic $q$ and for roots of unity. Now, let us assume that $q=e^{\pi i / m \varkappa}$, as in Section 3 .

Conjecture 7.6. The inner product on $\operatorname{Hom}_{\mathcal{C}(\mathfrak{g}, \varkappa)}\left(V_{\lambda}, V_{\mu} \otimes V_{\nu}\right)$ so defined is positive definite.

In the simplest case $\mathfrak{g}=\mathfrak{s l}_{2}$ this can be checked directly. In the general case, the answer is not known.

\section{ACKNOWLEDGMENTS}

I'd like to expresses my deep gratitude to my advisor Igor Frenkel for his guidance and encouragement. In particular, it is from his course that I first learned the structure of modular tensor category related to representations of quantum groups, as well as many other structures appearing in this paper.

Special thanks are due to Pavel Etingof. This paper continues the ideas introduced in a series of our joint papers, and the importance of discussions with Pavel for this paper cannot be overestimated.

Also, I'd like to thank Ivan Cherednik, Thomas Kerler, David Kazhdan, George Lusztig and Stephen Sawin for stimulating discussions and Harvard Mathematics department for its hospitality during my work.

Financial support was provided by Alfred P. Sloan dissertation fellowship.

\section{REFERENCES}

[A] Andersen, H. H., On tensor products of quantized tilting modules, Com. Math. Phys. 149 (1992), 149-159. MR 94b:17015

[AI] Askey, R. and Ismail, M.E.H., A generalization of ultraspherical polynomials, Studies in Pure Mathematics (P. Erdös, ed.), Birkhäuser, 1983, pp. 55-78. MR 87a:33015

[AP] Andersen, H. H. and Paradowski, J., Fusion categories arising from semisimple Lie algebras, Com. Math. Phys 169 (1995), 563-588. CMP 95:11

[Ch] Cherednik, I., Macdonald's evaluation conjectures and difference Fourier transform, preprint, May 1995, q-alg/9412016. CMP 96:02

[Dr1] Drinfeld, V.G., Quantum groups, Proc. Int. Congr. Math., Berkeley, 1986, pp. 798-820. MR 89f: 17017

[Dr2] _ , On almost cocommutative Hopf algebras, Leningrad Math.J. 1 (2) (1990), 321342. MR 91b: 16046

[EK1] Etingof, P.I. and Kirillov, A.A., Jr, A unified representation-theoretic approach to special functions, Functional Anal. and its Applic. 28 (1) (1994), 91-94. MR 95h:33010 
[EK2] , Macdonald's polynomials and representations of quantum groups, Math. Res. Let. 1 (1994), 279-296. CMP 95:04

[EK3] _ Representation-theoretic proof of inner product and symmetry identities for Macdonald's polynomials, hep-th/9410169, to appear in Comp. Math. (1995).

[F] Finkelberg, M., Fusion categories, Ph.D thesis, Harvard Univ. (1993), (to appear in GAFA).

[GK] Gelfand, S. and Kazhdan, D., Examples of tensor categories, Invent. Math. 109 (1992), 595-617. MR 93m:20057

[Kac] Kac, V.G., Infinite-dimensional Lie algebras, 3rd ed., Cambridge Univ. Press, 1990. MR 92k:17038

[Kas] Kassel, C., Quantum groups, Springer, New York, 1995. CMP 95:09

[KL1] Kazhdan, D. and Lusztig, G., Tensor structures arising from affine Lie algebras. I, J. of AMS 6 (1993), 905-947. MR 93m:17014

[KL2] - Tensor structures arising from affine Lie algebras. II, J. of AMS 6 (1993), 9491011. MR 93m:17014

[KL3] - Tensor structures arising from affine Lie algebras. III, J. of AMS 7 (1994), 335381. MR 94g:17048

[KL4] - Tensor structures arising from affine Lie algebras. IV, J. of AMS 7 (1994), 383453. MR 94g: 17049

[Ke] Kerler, T., Mapping class group actions on quantum doubles, Comm. Math. Phys. 168 (1995), 353-388. CMP 95:10

[KR] Kirillov, A.N. and Reshetikhin, N.Yu., q-Weyl group and a multiplicative formula for universal R-matrices, Comm. Math. Phys. 134 (1990), 421-431. MR 92c:17023

[L1] Lusztig, G., Modular representations and quantum groups, Contemp. Math., vol. 82, AMS, Providence, 1989, pp. 52-77. MR 90a:16008

[L2] , Finite-dimensional Hopf algebras arising from quantized universal enveloping algebras, J. of AMS 3 (1990), 257-296. MR 91e:17009

[L3] Quantum groups at roots of 1, Geom. Dedicat. 35 (1990), 89-113. MR 91j:17018

[L4] - On quantum groups, J. of Algebra 131 (1990), 466-475. MR 91j:17019

[L5] - Introduction to quantum groups, Birkhäuser, Boston, 1993. MR 94m:17016

[L6] Monodromic systems on affine flag manifolds, Proc. R. Soc. Lond. A 445 (1994), 231-246. MR 95m:20049

[L7] _ Canonical bases arising from quantized enveloping algebras. II, Progr. Theor. Phys. Suppl. 102 (1990), 175-201. MR 93g:17019

[LS] Levendorskii, S.Z. and Soibelman, Ya. S., Some applications of quantum Weyl groups, Jour. Geom. Phys. 7 (2) (1990), 241-254. MR 92g:17016

[Lyu] Lyubashenko, V., Modular transformations for tensor categories, J. Pure Appl. Algebra 98 (1995), 279-327. CMP 95:10

[M1] Macdonald, I.G., A new class of symmetric functions, Publ. I.R.M.A. Strasbourg, 372/S20, Actes 20 Séminaire Lotharingien (1988), 131-171.

[M2] Orthogonal polynomials associated with root systems, preprint (1988).

[Mac] MacLane, S., Categories for working mathematician, Graduate Texts in Mathematics, vol. 5, Springer-Verlag, New York, 1971. MR 50:7275

[MS1] Moore, G., Seiberg, N., Classical and quantum conformal field theory, Com. Math. Phys. 123 (1989), 177-254. MR 90e:81216

[MS2] Lectures on RCFT, Superstrings '89 (Proc. of the 1989 Trieste Spring School) (M. Green et al, eds.), World Sci., River Edge, NJ, 1990, pp. 1-129. MR 93m:81133a

[MS3] , Polynomial equations for rational conformal field theories, Phys. Let. B212 (1988), 451-460. MR 89m:81155

[MS4] _ Naturality in conformal field theory, Nucl. Phys. B313 (1989), 16-40. MR 90f: 81119

[RT1] Reshetikhin, N. and Turaev, V., Ribbon graphs and their invariants derived from quantum groups, Comm. Math. Phys. 127 (1990), 1-26. MR 91c:57016

[RT2] , Invariants of 3-manifolds via link polynomials and quantum groups, Inv. Math. 103 (1991), 547-597. MR 92b:57024

[Tu] Turaev, V., Quantum invariants of knots and 3-manifolds, W. de Gruyter, Berlin, 1994. MR 95k:57014 
[Vaf] Vafa, C., Towards classification of conformal theories, Phys. Lett. B 206 (1988), 421-426. MR 89k:81178

[We] Wenzl, H., Quantum groups and subfactors of type B, C, and D, Comm. Math. Phys. 133 (1990), 383-433. MR 92k:17032

Department of Mathematics, Massachusetts institite of Technology, Cambridge, Massachusetts 02139

E-mail address: kirillov@math.mit.edu, http://web.mit.edu/kirillov/www/home.html 